\title{
Experimental and theoretical insights into the structure of tellurium chloride glasses
}

Lila Bouëssel Du Bourg ${ }^{1}$, Eric Furet ${ }^{1, *}$, Alicia Lecomte ${ }^{1,2}$, Laurent Le Pollès ${ }^{1}$, Shinji Kohara ${ }^{3}$, Chris J. Benmore ${ }^{4}$, Eugène Bychkov ${ }^{5}$, David Le Coq ${ }^{2, *}$

${ }^{1}$ Institut des Sciences Chimiques de Rennes, UMR 6226 CNRS, Ecole Nationale Supérieure de Chimie de Rennes, 35708 Rennes, France

${ }^{2}$ Institut des Sciences Chimiques de Rennes, UMR 6226 CNRS, Equipe Verres et Céramiques, 35042 Rennes, France

${ }^{3}$ Synchrotron X-ray Group, Light/Quantum Beam Field Research Center for Advanced Measurement and Characterization, NIMS, 1-1-1 Kouto, Sayo-cho, Sayo-gun, Hyogo 6795148, Japan

${ }^{4}$ X-ray Science Division, Advanced Photon Source, Argonne National Laboratory, Argonne, Illinois 60439, USA

${ }^{5}$ Laboratoire de Physico-Chimie de l'Atmosphère - EA 4493 Université du Littoral-Côte d'Opale - 189A Av. M. Schumann, 59140 Dunkerque, France

Corresponding author email : eric.furet@ensc-rennes.fr 


\begin{abstract}
The structure of the binary chalcohalide glasses $\mathrm{Te}_{1-x} \mathrm{Cl}_{x}(0.35 \leq x \leq 0.65)$ is considered by combining experimental and theoretical results. The structural network properties are influenced by a competition between ionic and covalent bonding in such glasses. At first, a focus is placed on the detailed information available by using the complementary Highenergy X-ray and the neutron diffractions in both the reciprocal and real spaces. The main characteristic suggested by the structure factors $S(Q)$ concerns the presence of three length scales in the intermediate range order. The total correlation function $T(r)$ let also suppose that the structure of these glasses is more complicated than Te-chain fragments with terminal $\mathrm{Cl}$ as demonstrated in crystalline $\mathrm{Te}_{3} \mathrm{Cl}_{2}$. Molecular dynamics simulations were subsequently performed on $\mathrm{Te}_{3} \mathrm{Cl}_{2}$ and $\mathrm{Te}_{2} \mathrm{Cl}_{3}$, and coupled with the experimental data, a highly reticulated network of chalcogen atoms, with a fair amount of chlorine atoms bonded in a bridging mode is proposed. The simulations clearly lead to a glass description that differs markedly from the simple structural model based on only Te atom chains and terminal Cl atoms. Solid-state NMR experiments and NMR parameters calculations allowed to validate the presence of Te highly coordinated with chlorine in these glasses.
\end{abstract}




\section{Introduction}

Chalcogenide glasses are known to exhibit a wide range of tunable physical properties, and have therefore been the subject of numerous investigations over the past decades ${ }^{1,2,3}$. Among them, the family of so-called TeX glasses ${ }^{4}$, where X stands for Chlorine, Bromine or Iodine, which showed promising optical properties with a multiphonon absorption edge pushed away from the classical 8-12 $\mu \mathrm{m}$ atmospheric window, have received much less attention, most probably due to their low mechanical and thermal properties that precluded their applicability at the industrial-scale. Indeed, although the local structure of chalcogenide glasses has been extensively studied by X-ray and neutron diffraction for five decades, there is relatively little literature concerning specifically the subclass of tellurium chloride binary chalcohalide glasses. The structure of these materials is very interesting since chalcogens generally have covalent bonds ${ }^{5}$ and halides are predominantly ionic ${ }^{6}$. The first studies on the structure of glasses in $\mathrm{Te} / \mathrm{Cl}$ system were reported by Wells et al. ${ }^{7}$. Their ${ }^{125} \mathrm{Te}$ absorption and ${ }^{127} \mathrm{I}$ emission Mössbauer spectroscopic experiments led them to conclude that the structure of the specific composition $\mathrm{Te}_{3} \mathrm{Cl}_{2}$ may be described in terms of chain fragments. The originality of this chain model is the nature of the Te sites, where 3 different sites have been identified: a Te-site two-fold coordinated with Te nearest neighbors (NN) (A-site), a Te-site four-fold coordinated to a pair of both Te and Cl NN (B-site), and a Te-site identified as a chain terminating Te-site, having a Te and a $\mathrm{Cl}$ atoms as $\mathrm{NN}$ (C-site). It should be noted that the crystal structure of this very same stoichiometry was previously resolved by Kniep et al, and that it may have oriented, to some extent, the interpretation of the glass structure ${ }^{8}$ (Figure 1 ). On the theoretical side, the first attempt to rationalize the structure of the Te-Cl glasses, on the basis of quantum calculations, was performed by Suehara et al. using various molecular models ${ }^{9}$. Their results obtained on finite-sized systems do not led them to reconsider previous 
findings, i.e. a room temperature structural model based on Cl-terminated chain fragments. Other investigations by X-ray diffraction and Reverse Monte Carlo simulations have also been carried out for amorphous tellurium subhalides ${ }^{10}$. In this case, two specific compositions, $\mathrm{TeCl}_{2-x} \mathrm{I}_{x}(x=0.1$ and 0.5$)$, were studied and the authors assert that these glasses would comprise structural fragments that are present in both tellurium tetrahalides and in lowvalent tellurium subhalides.

In the present paper, macroscopic properties and structural studies by neutron and X-ray diffractions of several $\mathrm{Te}_{1-x} \mathrm{Cl}_{x}$ glasses, $0.35 \leq x \leq 0.70$, are presented. The combination of both techniques is extremely complimentary since the neutron coherent scattering lengths and the X-ray scattering form factors are significantly different for Te and Cl. Indeed, the neutron coherent scattering lengths $(b)$ are 5.80 and $9.58 \mathrm{fm}$, while the atomic numbers are 52 and 17 for $\mathrm{Te}$ and $\mathrm{Cl}$, respectively. Consequently, the neutron measurement is more sensitive to $\mathrm{Cl}$ $\left(b_{C l} / b_{T \mathrm{e}}=1.65\right)$ while $\mathrm{X}$-ray diffraction is more sensitive to Te $\left(f_{\mathrm{Te}} / f_{C l}=3.06\right)$. Moreover, the combination of the experimental results from large-scale neutron and synchrotron facilities, and also from ${ }^{125}$ Te solid-state nuclear magnetic resonance (NMR) spectroscopy, with $a b$ initio molecular dynamics (MD) should be very insightful in proposing an improved structural model for Te-Cl glasses. Indeed in recent years, researchers have gained many useful insights into the atomic scale structure of various binary or ternary chalcogenide glasses using various spectroscopic techniques (NMR, and/or diffraction, and/or Raman) and MD trajectories $^{11,12,13,14,15,16,17}$. In some cases it was shown that a combination of both the medium to long range and local probes are necessary to obtain a refined picture of the glassy structural organization. 


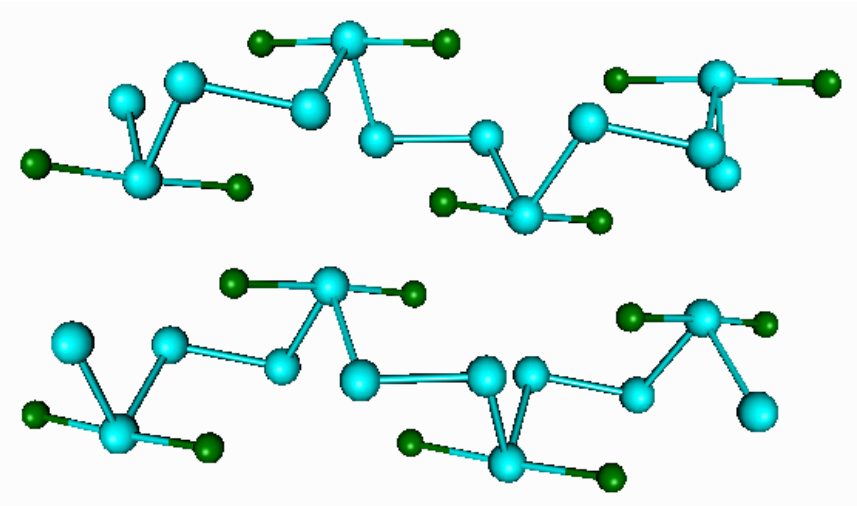

Figure 1. Representation of the crystalline structure of $\mathrm{Te}_{3} \mathrm{Cl}_{2}$ as resolved by Kniep et al. ${ }^{8}$. This is a portion of a 1x1x2 supercell. Te are in blue and $\mathrm{Cl}$ are in green.

\section{Material and methods}

\subsection{Experimental details}

Bulk glass samples of $\mathrm{Te}_{1-x} \mathrm{Cl}_{x}$, where $0.35 \leq x \leq 0.70$, were synthesised using pure elemental Te (Fluka, 99.999\%) and $\mathrm{TeCl}_{4}$ powder (Aldrich, 99\%). The starting materials were weighted in a glove box under an argon environment since $\mathrm{TeCl}_{4}$ is strongly sensitive to moisture, and placed in a silica tube before evacuation under vacuum and sealing. The silica tubes were slowly heated to $500^{\circ} \mathrm{C}$ in a rocking furnace for 24 hours before quenching the melt in water. The glassy nature of the samples was verified by X-ray diffraction.

The density $(d)$ was measured at room temperature by the Archimedes principle using toluene as the immersion medium and germanium as reference. Four measurements were performed for each composition using samples from 2 different syntheses.

Differential scanning calorimetric (DSC) curves of the prepared glasses were recorded using a thermal analysis apparatus (SEIKO) at a heating rate of $10 \mathrm{~K} \cdot \mathrm{min}^{-1}$. Typical sample weights were about $10 \mathrm{mg}$. Two measurements were performed for each composition. 


\subsection{Diffraction experiment details}

Neutron scattering (ND) experiments have been carried out on the hot source of the Orphée reactor at the Laboratoire Léon Brillouin (Saclay, France) using the 7C2 spectrometer with a $0.700 \AA$ incident wavelength ${ }^{18}$. Diffraction pattern were recorded with a 640-cell multidetector which provides an accurate determination of the spectra over the momentum transfer range of $0.4 \AA^{-1}<Q<16 \AA^{-1}$. Spectra were collected at room temperature for approximately 10 hours. Glass samples were contained in a vertical vanadium cell $(8 \mathrm{~mm}$ diameter, $0.01 \mathrm{~mm}$ thickness), the height of the neutron beam was $50 \mathrm{~mm}$. The neutron structure factors $S_{N}(Q)$ were obtained from the scattered intensities. Conventional data reduction procedures were applied for absorption, incoherent and multiple scattering effects. Inelastic effects were treated by a Placzek type correction. In order to obtain some data sets over a larger scattering vectors $\left(Q=40 \AA^{-1}\right)$, and consequently a better resolution in the real space, complementary time-of-flight ND experiments were also performed using the GLAD instrument ${ }^{19}$ at the intense pulsed neutron source (IPNS) in similar containers. The data analyses were done following standard procedures including the above corrections ${ }^{20,21}$.

High energy X-ray diffraction (HE-XRD) experiments of some glass compositions, $x=0.35$, 0.40 , and 0.50 , were carried out using an incident photon energy of $62 \mathrm{keV}$ on the horizontal two-axis diffractometer, built at the BL04B2, SPring-8 (Hyogo, Japan), using a wavelength $\lambda$ $=0.202 \AA$ A HE-XRD experiments of the richest-Te samples, $x=0.50,0.60$, and 0.65 were performed using the 11 ID-C diffractometer at the Advanced Photon Source (Argonne, USA), which provided a $1 \mathrm{~mm}^{2}$ beam at $115 \mathrm{keV}(\lambda=0.108 \AA)$. The intensity was measured by a cooled Ge detector as a function of $Q$ in the range of $0.3-32.5 \AA^{-1}$. The data were corrected for deadtime, absorption, inelastic (Compton) scattering and background using standard techniques $^{22}$ to obtain the X-ray structure factors $S_{X}(Q)$. 


\section{$2.3^{125}$ Te solid-state NMR spectroscopy}

${ }^{125}$ Te NMR spectra were measured on a $7 \mathrm{~T}$ Bruker spectrometer $\left(300 \mathrm{MHz}\right.$ for $\left.{ }^{1} \mathrm{H}\right)$ with a $83 \mathrm{kHz}$ RF Field strength. A single pulse sequence was used with a pulse duration of $1 \mu$ s and 4096 or 1024 scans (for $\mathrm{Te}_{40} \mathrm{Cl}_{60}$ and $\mathrm{Te}_{60} \mathrm{Cl}_{40}$ respectively) were accumulated. All spectra were recorded at a MAS rate of $10 \mathrm{kHz}$ in a 4 mm MAS probe. In the literature, a repetition delay of 20 seconds has been reported for $\mathrm{M}_{2} \mathrm{O}-\mathrm{TeO}_{2}\left(\mathrm{M}=\mathrm{Li}, \mathrm{Na}, \mathrm{K}, \mathrm{Rb}\right.$ and Cs) glasses ${ }^{23}$. In our case a repetition delay of 10 seconds was tested and it allowed us to obtain fully relaxed spectra. We used ZnTe as a secondary reference with a ${ }^{125}$ Te NMR spectrum peak at $-888 p p m$ relative to the primary reference $\left(\mathrm{CH}_{3}\right)_{2} \mathrm{Te}$ at $0 \mathrm{ppm}$.

\subsection{Molecular dynamics}

Based on experimental densities, of 4.62 g.cm ${ }^{-3}$ for $\mathrm{Te}_{0.60} \mathrm{Cl}_{0.40}$ and 3.96 g.cm ${ }^{-3}$ for $\mathrm{Te}_{0.40} \mathrm{Cl}_{0.60}$, we built two cubic simulation cells for each composition of $18.68 \AA$ and $18.28 \AA$ side length respectively, containing 200 atoms randomly distributed. On each cell, Car-Parrinello molecular dynamics simulations (MD) were performed using CPMD-3.17.1 package ${ }^{24,25}$, with Perdew-Burke-Ernzerhof (PBE) exchange and correlation functionals ${ }^{26}$, and Troullier-Martins norm-conserving pseudo-potentials ${ }^{27,28}$. The plane-wave basis set was expanded up to a kinetic energy cutoff of 30 Ry and calculations were carried out at the Gamma point of the Brillouin zone. In order to ensure Car-Parrinello energy conservation, we used a 7 a.u. (0.17 fs) integration step with a fictitious electronic mass of 850 a.u., and a Nosé-Hoover thermostat chain controlled the temperature ${ }^{29,30}$. In order to take into account Van der Waals interactions, we added empirical Grimme correction G06 ${ }^{31}$. Our molecular dynamics simulations began 
with a mixing stage at $800 \mathrm{~K}$ for $17 \mathrm{ps}$, then we reproduced a quenching process by reducing the temperature step by step, with a 17 ps $600 \mathrm{~K}$ plateau, a 25 ps $400 \mathrm{~K}$ plateau and a 20 ps 300K plateau where the glasses finished equilibrating. Statistical averages were taken from this last 300K plateau, representing more than 100000 steps.

After a CPMD optimization of final structures, we computed ${ }^{125}$ Te NMR parameters using the Gauge Including Projected Augmented Wave (GIPAW) formalism ${ }^{32,33}$ as implemented in the CASTEP package, version $16.1^{34}$. We used the PBE exchange and correlation functionals, onthe-fly generated ultrasoft pseudopotentials and an energy cutoff of $350 \mathrm{eV}$ after checking it ensured a convergence $<2$ ppm for chemical shift and chemical anisotropy. A 3x3x3 k-point grid was used to sample the Brillouin zone. GIPAW calculations give access to absolute shielding tensors $(\sigma)$ that are diagonalized to obtain their eigenvalues. A combination of these values gives access to NMR parameters through the equations:

$$
\begin{gathered}
\sigma_{i s o}=\frac{1}{3}\left(\sigma_{x x}+\sigma_{y y}+\sigma_{z z}\right) \\
\sigma_{\text {aniso }}=\sigma_{\mathrm{zz}}-\sigma_{\text {iso }} \\
\eta=\frac{\sigma_{y y}-\sigma_{x x}}{\sigma_{\text {aniso }}}
\end{gathered}
$$

, where in the Haeberlen convention ${ }^{35},\left|\sigma_{z z}-\sigma_{i s o}\right| \geq\left|\sigma_{z z}-\sigma_{i s o}\right| \geq\left|\sigma_{z z}-\sigma_{i s o}\right|$. Afterwards, we obtain isotropic chemical shift with the relation: $\partial_{i s o}=\sigma_{r e f}-c \sigma_{i s o}$ where, with a linear regression on ten crystals, $\sigma_{r e f}$ has been evaluated at $2815 \mathrm{ppm}$ and $c$ at 0.96 . Finally, spectra simulation has been produced using Simpson software that takes into account all previous NMR parameters to emulate the experimental spectra ${ }^{36}$. 


\section{Results and discussion}

Structural information can be obtained from macroscopic characterisations of the density $(d)$ and glass transition temperatures $(\mathrm{Tg})$, even if the data exhibit radical changes as a change in slope or threshold behaviour ${ }^{37,38,39}$. The evolution of these parameters is plotted in Figure 2 for several $\mathrm{Te}_{(1-x)} \mathrm{Cl}_{x}$ glasses. The evolution of $d$ and $\mathrm{Tg}$ in chloride glasses suggests that the structure doesn't change drastically with increasing $\mathrm{Cl}$ content. The linear decrease of $d$ as $x$ increases can be directly correlated to the difference in atomic masses between Te and $\mathrm{Cl}$. Besides, the extrapolation to $x=0$ give a density close to the density of the amorphous tellurium equal to 6.2 g. $\mathrm{cm}^{-3}$ confirming the linear evolution. In comparison, the density of $\mathrm{Te}_{(1-\mathrm{y})} \mathrm{Se}_{\mathrm{y}}$ exhibit a similar trend, which is correlated to a chain-like structure ${ }^{40}$. The composition dependence of $\mathrm{Tg}$ also exhibits a decreasing monotonic tendency, which indicates that there is no drastic change in the structure of these glasses. Moreover, $\mathrm{Tg}$ varies from $78^{\circ} \mathrm{C}(x=0.35)$ down to $45^{\circ} \mathrm{C}(x=0.7)$. These results are consistent with low dimensional networks such as chain-like or molecular-like structures.

Figure 3a-b shows the Faber-Ziman structure factors of $\mathrm{Te}_{1-x} \mathrm{Cl}_{x}$ glasses obtained in both the neutron (a) and X-ray (b) diffraction experiments. Distinct oscillations are well discernable for scattering vectors $Q$ up to $12 \AA^{-1}$ and $20 \AA^{-1}$ in the neutrons and X-ray scattering patterns, respectively. The two types of experiments show different features as a function of composition, notably in the low $Q$ region. Figure 4 focuses on this low $Q$ part and reveals very interesting characteristics. Usually, the binary chalcogenide glasses exhibit a more or less expressive first sharp diffraction peak (FSDP) in the region $1.0 \AA^{-1}<Q_{1 a}<1.5 \AA^{-1}$, but the $S(Q)$ of these samples clearly exhibit a second peak at lower $Q$-values, $Q_{0} \sim 0.6 \AA^{-1}$, and the $S_{X}(Q)$ also possesses a third peak at slightly higher $Q$-values, $Q_{1 b} \sim 1.6 \AA^{-1}$ (Figure $4 a$ ). 


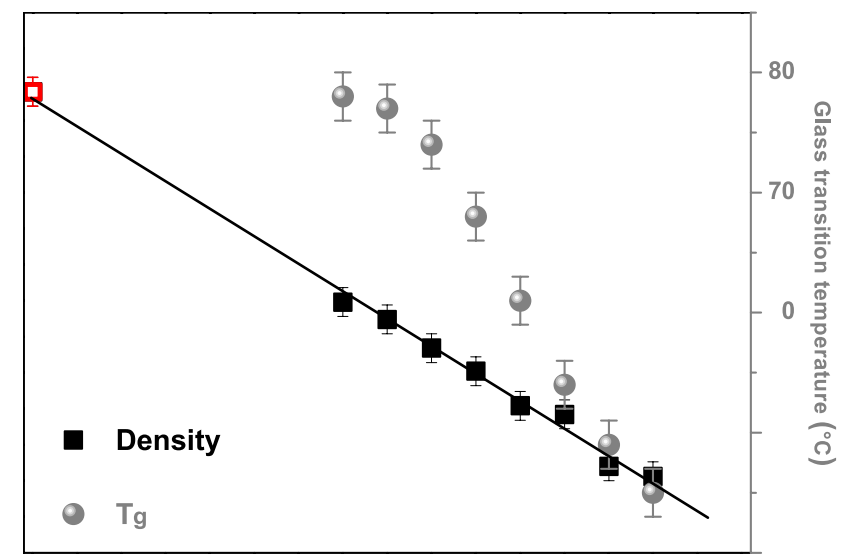

Figure 2. Dependences of the density, $d$ (black squares), and the glass transition temperature, $T g$ (grey circles) as a function of chlorine atomic content $x$. The density of amorphous tellurium is also plotted (empty red square) to underline the linear evolution of the density of the glasses.
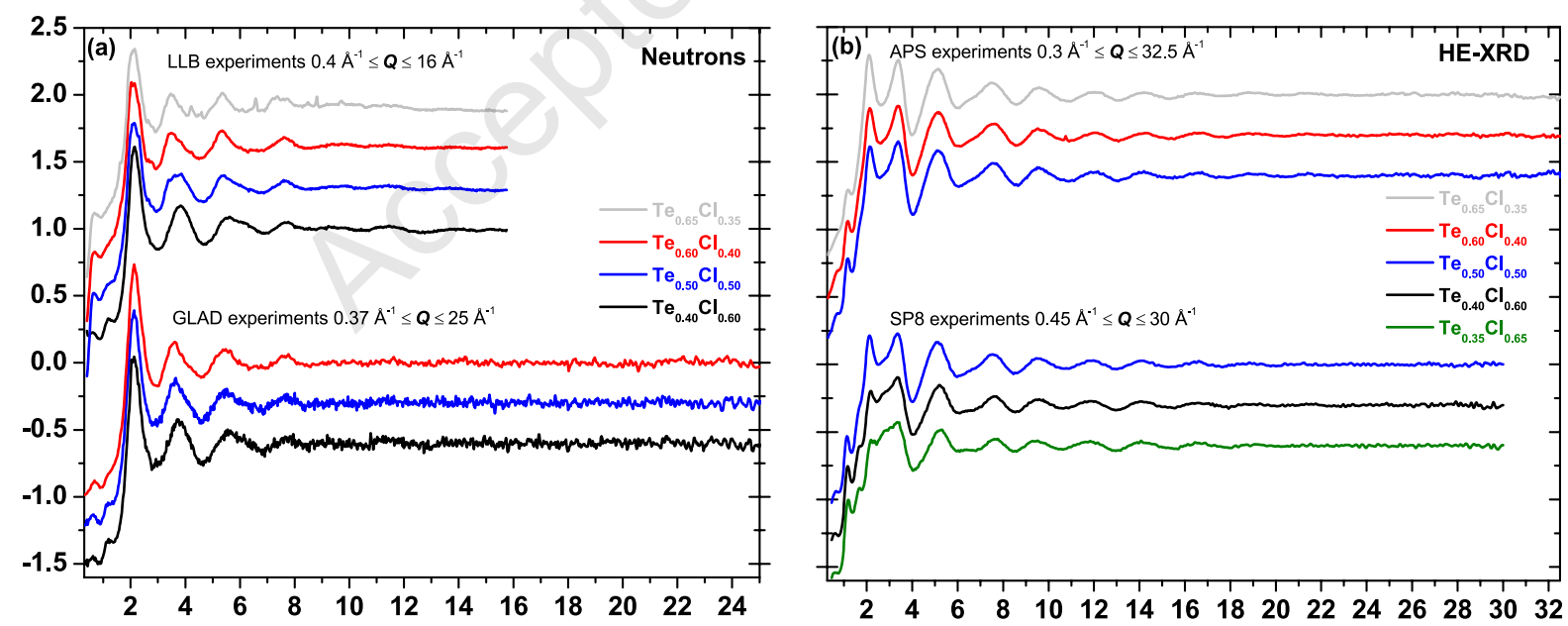

Figure 3. Faber-Ziman structure factors of $\mathrm{Te}_{(1-\mathrm{x})} \mathrm{Cl}_{\mathrm{x}}$ glasses, with $0.65 \leq x \leq 0.35$ : (a) neutron experiments performed at LLB (reactor) and GLAD (pulsed neutrons) and (b) high-energy X-ray experiments performed both at APS and SP8. 
It is known that the FSDP reflects intermediate-range ordering (IRO) in glasses at a characteristic periodicity given by $L_{i}=2 \pi / Q_{i}$. In order to extract the parameters of the FSDP, a Voigt function was used to approximate the background underneath it, allowing the prepeaks to be isolated and fitted with a Gaussian as exhibited in Figure 4a for the specific composition $\mathrm{Te}_{0.40} \mathrm{Cl}_{0.60}$. The first pre-peak at $Q_{0} \sim 0.6 \AA^{-1}$, corresponding to $L_{0} \sim 10 \AA$, is increasing with the $\mathrm{Cl}$ content. It is also proportionally more intense in neutron than in X-ray data (Figures 3 and 4a). These features indicate that the chlorine interactions have a high contribution on this length-scale. The second pre-peak at $Q_{1 a} \sim 1.15 \AA^{-1}$, corresponds to a relative invariant length $L_{1 a} \sim 5.5 \AA$. Its area increases with $\mathrm{Cl}$ content and is more intense in X-ray data. The last pre-peak located around $1.6 \AA^{-1}$ is invisible in neutron experiments and tends to disappear with an increase of $x$ indicating that it is related to Te. Its corresponding periodicity of $L_{1 b} \sim 3.9 \AA$ is relatively constant. At last, the areas of the peaks at $Q_{0}$ and $Q_{1 a}$ increase with the $\mathrm{Cl}$ content in both the neutron and X-ray patterns, while the peak area at $Q_{1 b}$ decreases (Figure 4b). With regard to the peak positions, they are relatively constant for $Q_{1 a}$ and $Q_{1 b}$, and decrease for $Q_{0}$ as $x$ is increased (Figure 5).

Multiple pre-peaks at low $Q$ appear to be a very rare phenomenon in inorganic glasses. In this specific context, we assume that only pre-peaks having anomalous temperature, pressure and composition dependences as the First Sharp Diffraction Peak (FSDP) and, consequently, different from all other peaks in the structure factor of a glass or liquid ${ }^{41,42,43}$, should be taken into account. Up to date, only a few alkali silicate glasses have shown a transformation of the intense $\mathrm{SiO}_{2}$-related FSDP into a weak poorly resolved broad feature consisting of two or three contributions ${ }^{44}$. This complex feature was found to be dependent on alkali type and concentration in the glass. In vitreous chalcogenides, the FSDP is usually associated with intermediate-range ordering of the network-forming cations (NFC), i.e., Ge in germanium 
chalcogenide glasses ${ }^{45,46}$. The NFC periodicity, $L_{N F C}=2 \pi / Q_{F S D P}$, reflects the ring statistics in these network glasses ${ }^{47,48}$. The Te-Cl compositions appear to be significantly different from the widely studied 2D or 3D chalcogenide network binaries going from extended 1D tellurium chains to small diatomic molecules $\mathrm{Cl}_{2}$ passing through $\mathrm{Te}_{3} \mathrm{Cl}_{2}$ chains and $\mathrm{Te}_{4} \mathrm{Cl}_{16}$ tetramers in crystalline state ${ }^{8,49}, \mathrm{Te}_{2} \mathrm{Cl}_{8}$ dimers in liquid ${ }^{50}$ and $\mathrm{TeCl}_{4}$ monomers ${ }^{51,52}$ in gas phases. A complicated hierarchy of the Te-Cl structural organization on atomic scale (2-fold and 4-fold coordinated tellurium atoms, terminal and bridging chlorine species) implies different intermediate-range scales for constituent units.
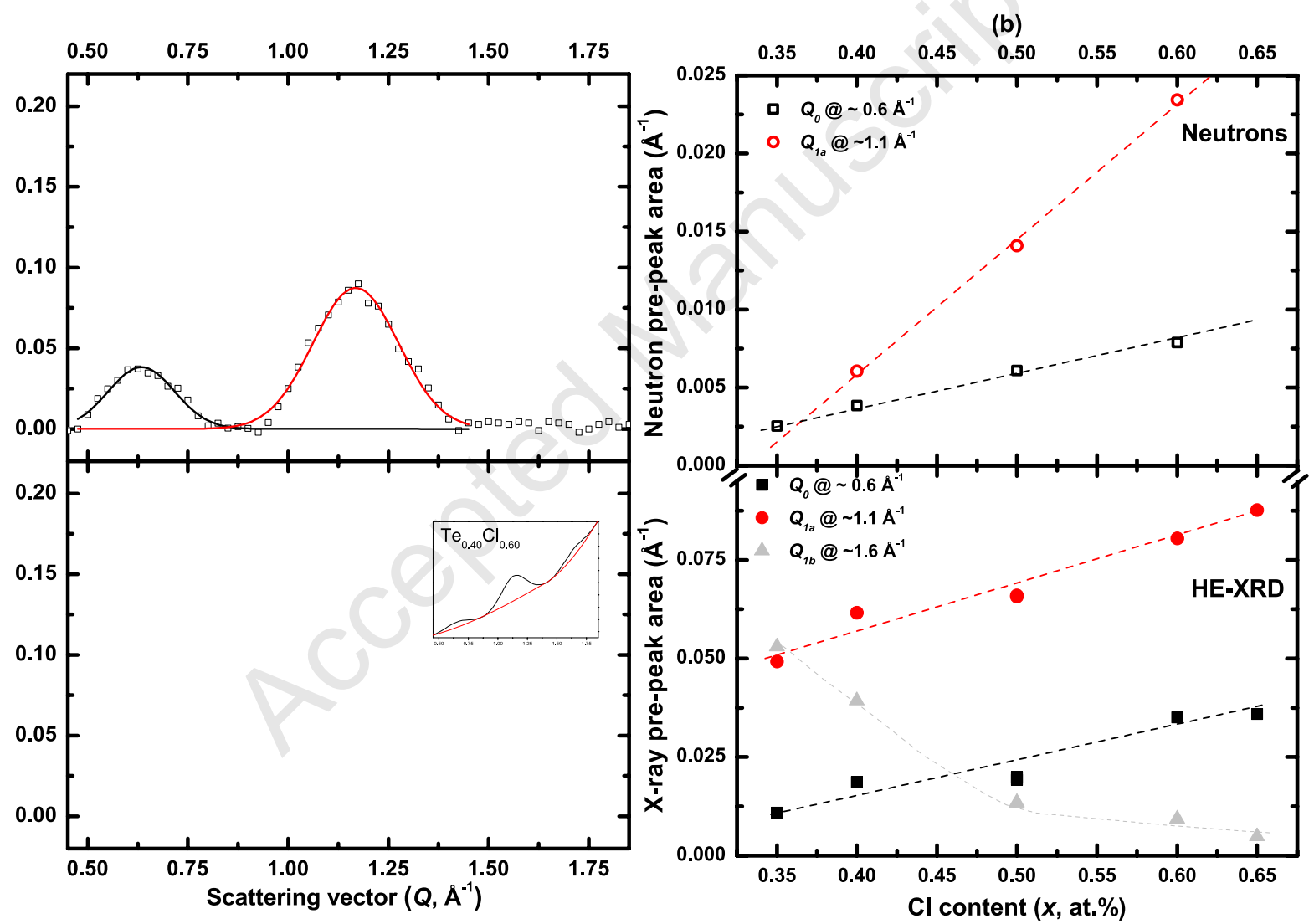

Figure 4. (a) Typical pre-peaks at $Q_{0}, Q_{1 a}$, and $Q_{1 b}$ in $\mathrm{Te}_{0.40} \mathrm{Cl}_{0.60}$ isolated from the HE-XRD and neutrons structure factors. (b) Evolution of the three pre-peak areas corresponding to the peak positions at $Q_{0}, Q_{1 a}$, and $Q_{1 b}$ shown as a function of $\mathrm{Cl}$ content from neutron (up) and Xray (down) diffraction data. The dash lines represent a guide for the eyes. 


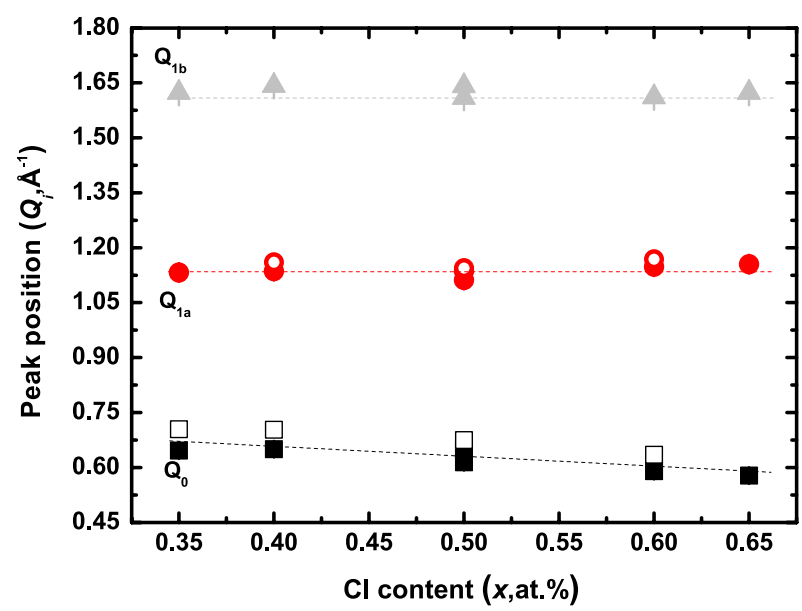

Figure 5. Evolution of the three pre-peak positions $\left(Q_{0}, Q_{1 a}\right.$, and $\left.Q_{1 b}\right)$ as a function of $\mathrm{Cl}$ content, taken from neutron (empty symbols) and X-ray (full symbols) diffraction $S(Q)$. The dash lines represent a guide for the eyes.

In order to corroborate the above assumptions about the origin of the multiple pre-peaks, first order difference structure factors $\Delta S(Q)$ without Te-Te, Te-Cl or Cl-Cl correlations (Figure 6) are calculated using a combination of neutron and high-energy X-ray diffraction data. For example, removing the Te-Te correlations can be achieved through the following equation:

$\Delta S_{-\mathrm{TeTe}}(Q)-1=\left\{\left[S_{X}(Q)-1\right]-\frac{W_{\mathrm{TeTe}}^{X}(Q)}{W_{\mathrm{TeTe}}^{N}}\left[S_{N}(Q)-1\right]\right\} \frac{W_{\mathrm{TeCl}}^{X}(Q)}{W_{\mathrm{TeCl}}^{X}(Q)-\frac{W_{\mathrm{TeTe}}^{X}(Q)}{W_{\mathrm{TeTe}}^{N}} W_{\mathrm{TeCl}}^{N}}$,

where $S_{X}(Q)$ and $S_{N}(Q)$ are the Faber-Ziman X-ray and neutron structure factors, $W_{a b}^{X}(Q)$ are the $Q$-dependent X-ray weighting factors, and $W_{a b}^{N}$ are the neutron weighting coefficients. The second term in Eq. 1 normalizes the difference structure factor $\Delta S(Q)$ to the original (before subtraction) Te-Cl correlations. As a consequence, it is clear that the pre-peak at $Q_{0} \approx$ $0.6 \AA^{-1}$ is mostly related to Te-Cl correlations. Next, the most intense feature at $Q_{1 a} \approx 1.15 \AA^{-1}$ has two contributions: Te-Te (major) and Te-Cl (minor). Finally, the $Q_{1 b} \approx 1.6 \AA^{-1}$ peak 
represents essentially the Te-Te correlations. No contribution to any low-Q pre-peaks was found for $\mathrm{Cl}-\mathrm{Cl}$ atomic pairs.

The $1.1 \AA^{-1}$ and $1.6 \AA^{-1}$ pre-peaks, reflecting IRO in Te-Cl glasses, do not contradict the suggested chain-like structure of the vitreous alloys and are reminiscent of the IRO in the crystalline reference, monoclinic $\mathrm{Te}_{3} \mathrm{Cl}_{2}$ (space group $\mathrm{P} 2_{1} / \mathrm{n}$ ) ${ }^{8}$. The characteristic periodicity $L_{1 \mathrm{a}} \approx 5.5 \AA$ could correspond to both intra- and interchain Te-Te correlations of 4-fold coordinated Te species having $2 \mathrm{Te}$ and $2 \mathrm{Cl}$ nearest neighbors as suggests the dual nature of the $1.15 \AA^{-1}$ pre-peak. In monoclinic $\mathrm{Te}_{3} \mathrm{Cl}_{2}$, the corresponding Te-Te distances change between $5.64 \AA$ (shortest intrachain separation) and 4.8-5.6 $\AA$ (shortest interchain distances). The $1.6 \AA^{-1}$ pre-peak, also found in amorphous tellurium ${ }^{43}$, with the corresponding characteristic periodicity $L_{1 \mathrm{~b}} \approx 3.9 \AA$ reflects both intra- and interchain Te-Te correlations in tellurium helices, which are given at 4.1-4.2 $\AA$ in $c-\mathrm{Te}_{3} \mathrm{Cl}_{2}$. The lower Te-Te chain-like periodicity, $L_{1 b} \approx 3.9 \AA$, is also typical for flexible chalcogen structures characterized by weak and broad FSDPs, whose amplitude and position are best modeled by a random packing of chalcogen dimers, e.g. $\mathrm{Te}_{2}$ placed in the centers of spheres of diameter $3.35 \AA$, using the Perkus-Yevick formalism ${ }^{43}$. In contrast, the largest periodicity, $L_{0} \approx 10 \AA$, originating from the $0.6 \AA^{-1}$ pre-peak mostly related to Te-Cl correlations, does not have a simple explanation. Most probably, this intermediate-range scale indicates the formation of rather large structural fragments containing periodically arranged chlorinated Te species. But, the question of exact positioning the chlorinated entities in Te-Cl glasses remains open and needs further studies. 


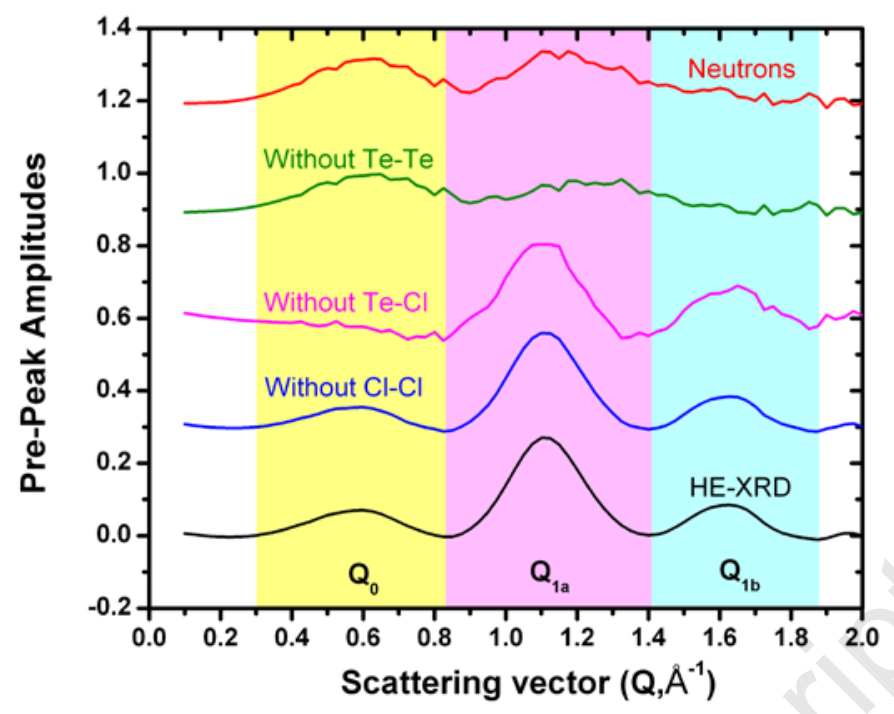

Figure 6. The multiple pre-peaks at $Q_{0} \approx 0.6 \AA^{-1}, Q_{1 a} \approx 1.1 \AA^{-1}$, and $Q_{1 b} \approx 1.6 \AA^{-1}$ highlighted by yellow, magenta and blue, respectively, have been isolated from either the X-ray $S_{X}(Q)$ (black) and neutron $S_{N}(Q)$ (red) structure factors, or from the difference structure factors $\Delta S(Q)$. These difference curves without Cl-Cl (blue), Te-Cl (magenta) or Te-Te (green) correlations are shown for the $\mathrm{Te}_{0.50} \mathrm{Cl}_{0.50}$ glass.

In the real space, this work focuses only on two compositions, namely $\mathrm{Te}_{0.60} \mathrm{Cl}_{0.40}\left(\mathrm{Te}_{3} \mathrm{Cl}_{2}\right)$ and $\mathrm{Te}_{0.40} \mathrm{Cl}_{0.60}\left(\mathrm{Te}_{2} \mathrm{Cl}_{3}\right)$, which correspond to the compositions also investigated by molecular dynamics simulations. The total pair correlation functions $g(r)$ of both the neutron and X-ray experiments were obtained by a Fourier transformation of $S(Q)$ as follows:

$$
g(r)=1+\frac{1}{2 \pi^{2} \rho r} \int\left(S_{N}(Q)-1\right) Q \sin (Q r) d Q, \quad(\text { Eq. 2) }
$$

, where $\rho$ is the average number density of the samples. The corresponding total correlation functions $T(r)$, 


$$
T(r)=4 \pi r \rho g(r)
$$

are plotted in Figure 7. For both the $\mathrm{Te}_{3} \mathrm{Cl}_{2}$ and $\mathrm{Te}_{2} \mathrm{Cl}_{3}$ compositions, $T(r)$ is used to obtain the correlation distances $r$ and the local coordination numbers $C N_{\mathrm{ij}}$ in the systems using a leastsquare fitting of the experimental data to Gaussian functions (Table 1).
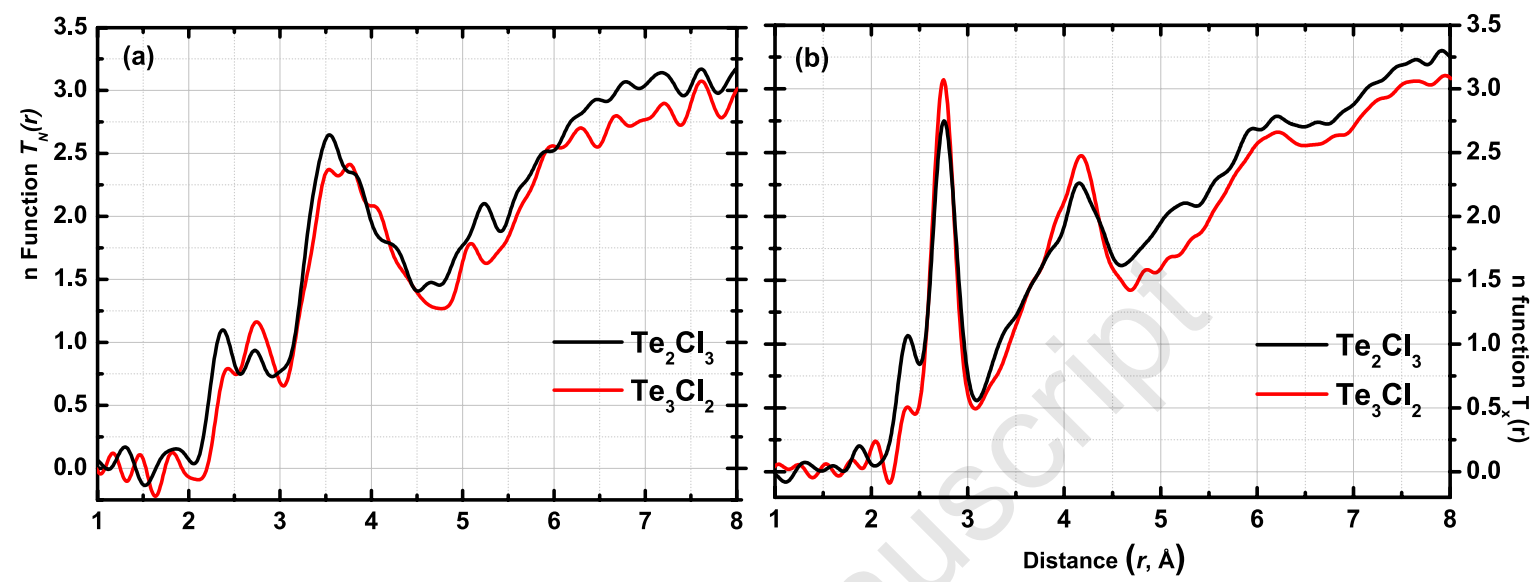

Figure 7. Faber-Ziman (a) neutron and (b) X-ray total correlation functions of $\mathrm{Te}_{2} \mathrm{Cl}_{3}$ (black) and $\mathrm{Te}_{3} \mathrm{Cl}_{2}$ (red) from which the experimental the coordination numbers $C N_{T e-T e}, C N_{T e-C l}$, and $\mathrm{CN}_{\mathrm{Cl}-\mathrm{Te}}$ were calculated.

First of all, we note that the shapes of the $T(r)$ functions are different for neutron and X-ray scattering. Even if the observable correlations at low distances are well defined, at this level it is difficult to establish the number of contributions present up to $3 \AA$. In order to solve this problem, a comparison of neutron total correlation functions $T_{N}(r)$ for glassy g- $\mathrm{Te}_{0.60} \mathrm{Cl}_{0.40}$ and its crystalline counterpart is shown in Figure 8 for two independent data sets with $Q_{\max }=$ $16 \AA^{-1}$ (reactor measurements) and $30 \AA^{-1}$ (pulsed neutron measurements). 


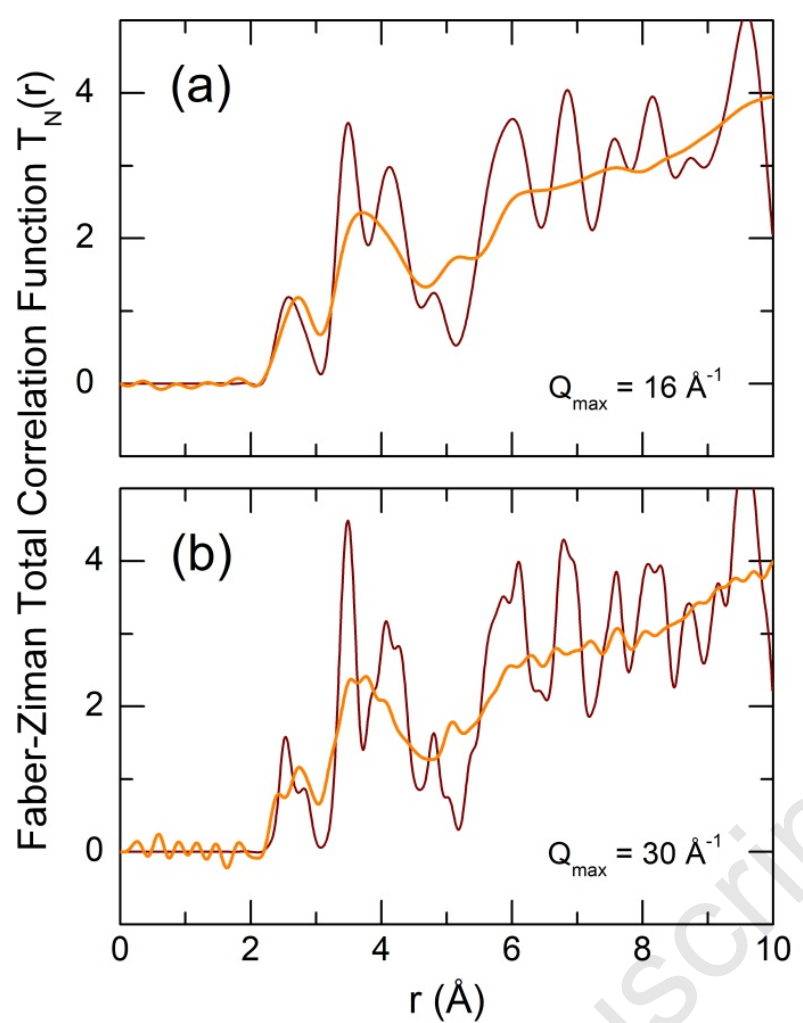

Figure 8. Neutron total correlation functions $T_{N}(r)$ for glassy g- $\mathrm{Te}_{3} \mathrm{Cl}_{2}$ (orange solid line) and monoclinic $\mathrm{Te}_{3} \mathrm{Cl}_{2}$, space group $P 2_{1} / n^{8}$ (brown line) for two different data sets with (a) $Q_{\max }=16 \AA^{-1}$ (reactor measurements) and (b) $Q_{\max }=30 \AA^{-1}$ (pulsed neutron measurements). The crystal data were obtained using the XTAL code ${ }^{53}$ for the above two $Q_{\max }$ values.

A higher resolution data $\left(30 \AA^{-1}\right)$ for $\mathrm{c}-\mathrm{Te}_{3} \mathrm{Cl}_{2}$ clearly shows a bimodal feature for the first neighbour correlations: (1) Te-Cl at $2.52 \pm 0.02 \AA$ and (2) Te-Te at $2.78 \pm 0.10 \AA$. The corresponding coordination numbers $C N_{\mathrm{Cl}-\mathrm{Te}}=1$ and $C N_{\mathrm{Te}-\mathrm{Te}}=2$ are related to terminal chlorine species, $\mathrm{Cl}_{t}$, associated with tellurium chains.

The first neighbour correlations in $\mathrm{g}-\mathrm{Te}_{60} \mathrm{Cl}_{40}$ show some similarities and differences compared to monoclinic c- $\mathrm{Te}_{3} \mathrm{Cl}_{2}$. The bimodal feature exhibits distinctly lower amplitude for the shorter $\mathrm{Te}^{-} \mathrm{Cl}_{t}$ correlations and more intense and broad peak at $r \approx 2.74 \AA$. A two-peak fitting of the feature yields $C N_{\mathrm{Cl}-\mathrm{Te}} \cong 0.6$ and $C N_{\mathrm{Te}-\mathrm{Te}}>2$. The small $\mathrm{Cl}_{t}$-Te coordination number suggests two contrasting hypotheses: (a) the presence of molecular chlorine $\mathrm{Cl}_{2}$ in the 
glasses, or (b) a simultaneous presence of terminal $\mathrm{Cl}_{t}$ and bridging $\mathrm{Cl}_{b}$ species coexisting with tellurium chains. The first suggestion is hardly to be realised from both chemical viewpoint (high chlorine reactivity) and the obtained experimental results. We did not observe a green chlorine vapour in the silica tubes during or after synthesis as well as the $\mathrm{Cl}-\mathrm{Cl}$ first neighbour correlations characteristic for molecular $\mathrm{Cl}_{2}, 1.95 \AA$ for liquid $\mathrm{Cl}_{2}{ }^{54}$ or $1.98 \pm 0.02$ $\AA$ for solid $\mathrm{Cl}_{2}{ }^{55,56}$.

The second hypothesis seems to be more realistic. First, terminal and bridging chlorine species are observed in many binary compounds, i.e., $\mathrm{Te}_{4} \mathrm{Cl}_{16}$ tetramers ${ }^{49,57}$ or $\mathrm{Ga}_{2} \mathrm{Cl}_{6}$ dimers ${ }^{58,59,60}$. The interatomic distances involving bridging $\mathrm{Cl}_{b}$ are always longer than those with $\mathrm{Cl}_{t}, 0.15 \AA \leq \Delta r \leq 0.40 \AA \AA^{49,54-57}$, where $\Delta r=r_{\mathrm{M}-\mathrm{Cl}_{b}}-r_{\mathrm{M}-\mathrm{Cl}_{t}}$. Consequently, the Te-Cl$b$ correlations overlap with Te-Te first neighbour distances at $\approx 2.8 \AA$.

Insufficiently resolved first neighbour feature makes a three-peak fitting challenging. Nevertheless, a high neutron/X-ray contrast for the Te-Cl glasses and an additional constraint related to the Cl-Te coordination numbers, i.e., $C N_{\mathrm{Cl}_{t}-\mathrm{Te}}=1$ and $C N_{\mathrm{Cl}_{b}-\mathrm{Te}}=2$, or, on the partial coordination number level, $2\left(1-C N_{\mathrm{Cl}_{t}-\mathrm{Te}}\right) \approx C N_{\mathrm{Cl}_{b}-\mathrm{Te}}$, allows a three-peak fitting to be performed. The derived results for $\mathrm{Te}_{3} \mathrm{Cl}_{2}$ and $\mathrm{Te}_{2} \mathrm{Cl}_{3}$ compositions are given in Table 1.

This first peak around $r_{1} \approx 2.4 \AA$ (Figure 7 ) attributed to $\mathrm{Cl}_{\mathrm{t}}$-Te first neighbours is consistent with the $\mathrm{Cl}$ and Te covalent radii being equal to $1.35 \AA$ and $0.99 \AA$, respectively. Also, similar Cl-Te bond length was also found in $\mathrm{Te}_{(1-\mathrm{x})} \mathrm{Cl}_{\mathrm{x}}$ liquids ${ }^{61}$. Higher the $\mathrm{Cl}$ content is in the composition, higher the $C N_{C l t-T e}$ is. At $r_{2} \approx 2.68 \AA$, corresponding to $\mathrm{Cl}_{\mathrm{b}}$-Te first neighbour correlation, the trend is reverse. The values given in Table 1 allow underlining that $\mathrm{Te}_{2} \mathrm{Cl}_{3}$, comparatively to $\mathrm{Te}_{3} \mathrm{Cl}_{2}$, possesses a higher $C N_{C l t-T e}$, a lower $C N_{C l b-T e}$, and a lower total $C N_{C l-}$ $T e$ in the two types of diffraction. The contribution at $r_{3} \approx 2.80-2.85 \AA$ is more intense both for the richest Te composition and in $T_{X}(r)$ comparatively to $T_{N}(r)$. Consequently, as mentioned 
above this correlation in the short-range order is related to Te-Te first neighbours, in good accordance with distances between two Te atoms given in literature for amorphous $\mathrm{Te}^{62}$ as well as for the Te-chain fragment in Te halide glasses ${ }^{7}$ and liquids ${ }^{61}$. The associated $C N_{T e-T e}$, lower than 2 is also logically higher for $\mathrm{Te}_{3} \mathrm{Cl}_{2}$ comparatively to $\mathrm{Te}_{2} \mathrm{Cl}_{3}$, and by taking into account the correlation Te-Cl, the total $\mathrm{CN}$ for Te reaches $\approx 2.6$ and $\approx 3.4$, respectively.

Table 1: Interatomic distances $r_{i}$, partial coordination numbers $C N_{C l-T e}$ (taking into account the terminal and bridging character of $\mathrm{Cl}$ ) and $C N_{T e-T e}$ derived from a three-peak fitting of the first neighbour feature in neutron $T_{N}(r)$ and X-ray $T_{X}(r)$ total correlation functions for the $\mathrm{Te}_{2} \mathrm{Cl}_{3}$ and $\mathrm{Te}_{3} \mathrm{Cl}_{2}$ glasses.

\begin{tabular}{|c|c|c|c|c|c|c|c|c|c|}
\hline & \multicolumn{5}{|c|}{ Cl-Te correlations } & D & \multicolumn{3}{|c|}{ Te- $\mathrm{X}$ correlations $(\mathrm{X}=\mathrm{Te}$ or $\mathrm{Cl})$} \\
\hline & \multicolumn{2}{|c|}{$\mathrm{Cl}_{\mathrm{t}}-\mathrm{Te}$} & \multicolumn{2}{|c|}{$\mathrm{Cl}_{\mathrm{b}}-\mathrm{Te}$} & \multirow{2}{*}{$\begin{array}{c}\mathrm{Cl}-\mathrm{Te} \\
\text { Total } C N_{\mathrm{Cl}-\mathrm{Te}} \\
\end{array}$} & \multicolumn{2}{|c|}{ Te-Te } & \multirow{2}{*}{$\begin{array}{c}\text { Te-Cl } \\
\text { Total } C N_{T e-C l} \\
\end{array}$} & \multirow{2}{*}{$\begin{array}{c}\text { Te-X } \\
\text { Total } C N_{T e-X} \\
\end{array}$} \\
\hline Composition & $r_{1}(\AA)$ & $C N_{\text {Clt-Te }}$ & $r_{2}(\AA)$ & $C N_{\text {Clb-Te }}$ & & $r_{3}(\AA$ & $C N_{T e-T e}$ & & \\
\hline \multicolumn{10}{|c|}{ NEUTRONS } \\
\hline $\mathrm{Te}_{2} \mathrm{Cl}_{3}$ & $2.37(1)$ & $0.78(10)$ & $2.68(2)$ & $0.45(10)$ & $1.23(20)$ & $2.87(5)$ & $1.52(15)$ & $1.82(15)$ & $3.34(30)$ \\
\hline $\mathrm{Te}_{3} \mathrm{Cl}_{2}$ & $2.42(2)$ & $0.56(10)$ & $2.68(3)$ & $0.74(10)$ & $1.30(20)$ & $2.87(5)$ & 1.72(15) & $0.87(15)$ & $2.59(30)$ \\
\hline \multicolumn{10}{|c|}{ HE-XRD } \\
\hline $\mathrm{Te}_{2} \mathrm{Cl}_{3}$ & $2.37(1)$ & $0.69(10)$ & $2.68(2)$ & $0.63(10)$ & $1.32(20)$ & $2.80(3)$ & $1.46(10)$ & $1.98(10)$ & $3.44(20)$ \\
\hline $\mathrm{Te}_{3} \mathrm{Cl}_{2}$ & $2.39(2)$ & $0.65(10)$ & $2.65(2)$ & $0.73(10)$ & $1.38(20)$ & $2.76(3)$ & $1.73(10)$ & $0.93(10)$ & $2.66(20)$ \\
\hline
\end{tabular}

In the medium-range order region, $3 \AA \leq r \leq 5 \AA, T_{X}(r)$ exhibits a decreasing contribution centered around $4.2 \AA$ with increasing $\mathrm{Cl}$ content. At the same time, a small contribution seems to grow in at $r \approx 3.4 \AA$. These features are corroborated in $T_{N}(r)$ since a shift towards lower distances is observed in this $r$-range. This broad asymmetric peak is assumed to be the result of two main contributions. At peak at $r \approx 4.2 \AA$, which is more distinct in the X-rays data, it corresponds to the Te-Te second neighbour intrachain or/and interchain correlations ${ }^{63}$. At peak at $r \approx 3.4 \AA$, which is more distinct in the neutron data, it is more heavily weighted towards $\mathrm{Cl}$ interactions. 
On the theoretical side, independent trajectories, for two Te-Cl glass compositions, namely $\mathrm{Te}_{2} \mathrm{Cl}_{3}$ and $\mathrm{Te}_{3} \mathrm{Cl}_{2}$, were simulated using ab initio molecular dynamics in order to produce in silico samples. The neutron and X-ray total radial distribution functions calculated from the corresponding 300K plateaus are given in Figure 9.
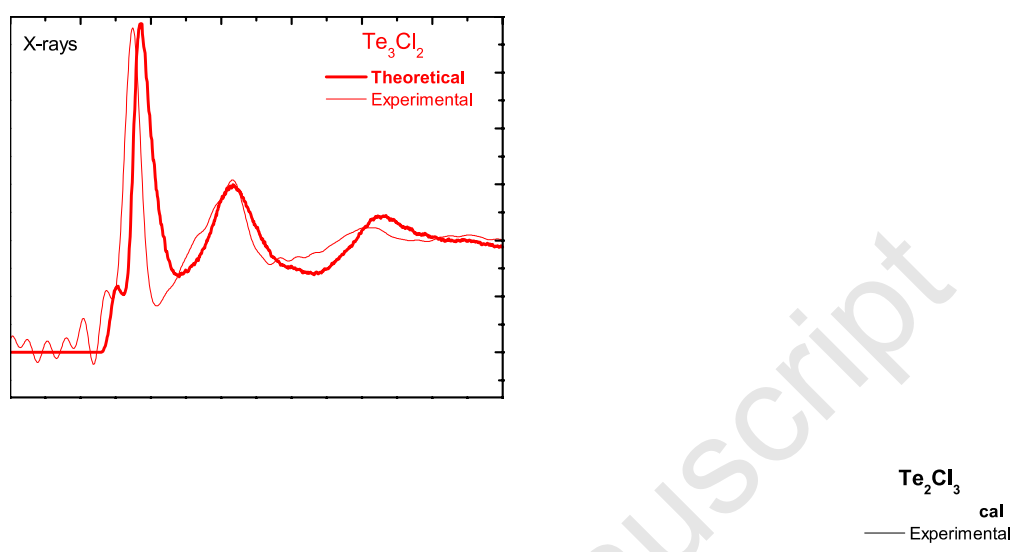

Figure 9. Experimental (thin line) and theoretical (bold line) X-Rays (left) and Neutrons (right) g(r), for $\mathrm{Te}_{2} \mathrm{Cl}_{3}$ (black) and $\mathrm{Te}_{3} \mathrm{Cl}_{2}$ (red).

In both cases, the agreement between the theoretical and experimental functions can be considered as good. Except for the low range part, i.e. below $2.5 \AA$, where the experimental radial distribution functions displays unphysical oscillations and a slight mismatch in peak positions, the relative peak intensities and the extrema positions are found to behave in a consistent way for the two compositions. It should be noted that the theoretical positions of the extremas are slightly shifted towards higher values with respect to the experimental ones, indicating therefore that the computed distances are a bit overestimated, due to the various 
approximations inherent to the calculations (density functional approximation, truncated basis sets,... ).

Turning to an analysis on the basis of the calculated partial radial distribution functions $\left(g_{i j}(r)\right)$, that were used to construct the $g(r)$ s, allows us to gain further insights into the structure of our glasses. Figure 10 shows these $g_{i j}(r)$ and their integrated areas $\left(n_{i j}(r)\right)$ from which theoretical coordination numbers $\left(C_{i j}\right)$ can be deduced (Table 2) and compared with experimental values.
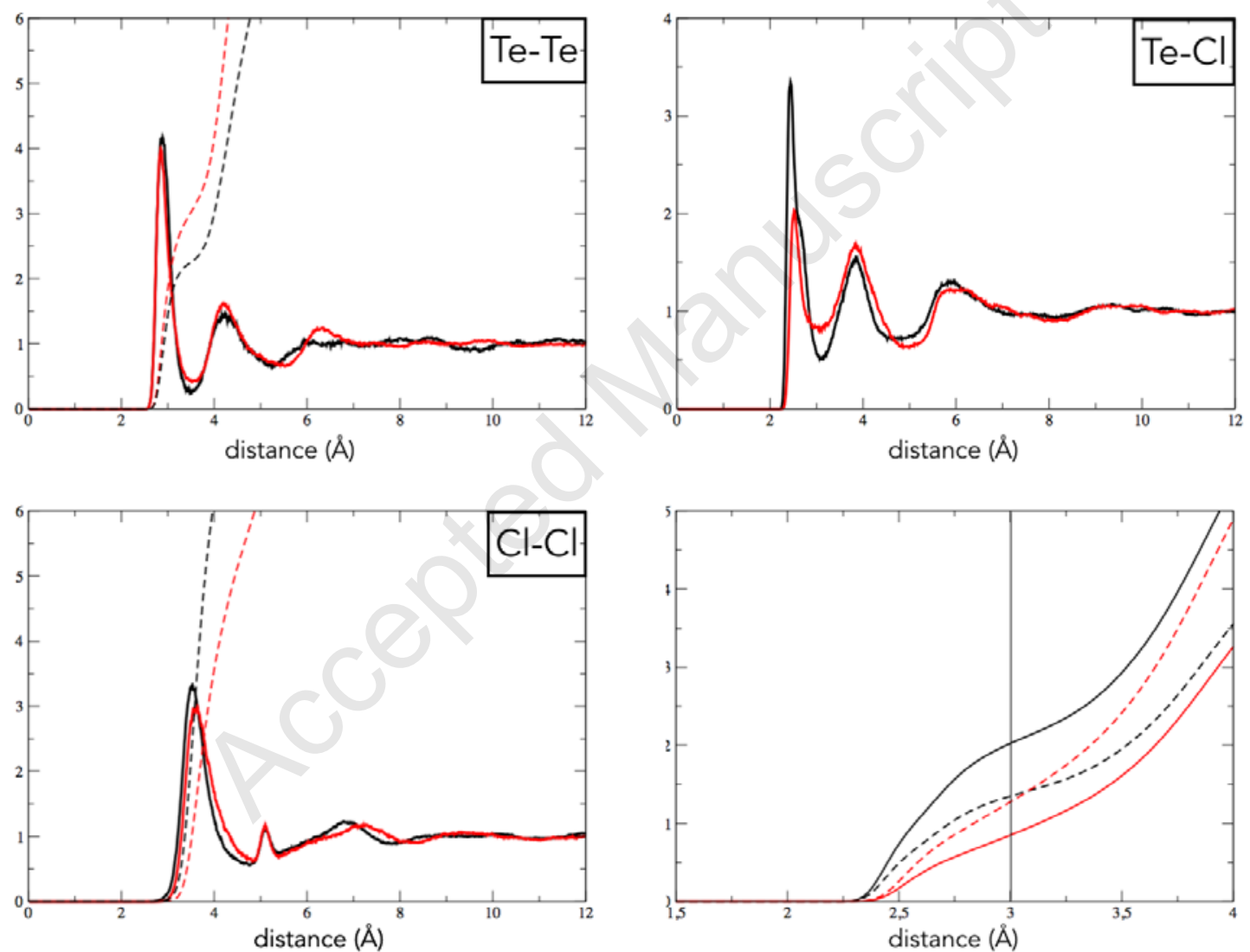

Figure 10. Theoretical $g_{\mathrm{TeTe}}(\mathrm{r})$ (top-left), $\mathrm{g}_{\mathrm{TeCl}}(\mathrm{r})$ (top-right), $\mathrm{g}_{\mathrm{ClCl}}(\mathrm{r})$ (bottom-left) and their respective integrated areas $n_{i j}(r)$ given by the dotted lines. $n_{\text {TeCl }}(r)$ and $n_{C I T e}(r)$ are at the bottom-right, respectively as the solid line and dotted line. The red color represents $\mathrm{Te}_{3} \mathrm{Cl}_{2}$ and the black is for $\mathrm{Te}_{2} \mathrm{Cl}_{3}$. 
Table 2. Coordination numbers for each composition, calculated from MD trajectories.

\begin{tabular}{|l|c|c|}
\hline Composition & $\mathrm{Te}_{3} \mathrm{Cl}_{2}$ & $\mathrm{Te}_{2} \mathrm{Cl}_{3}$ \\
\hline$C N_{\text {TeTe }}$ & 2.8 & 2.2 \\
\hline $\mathrm{CN}_{\text {TeCl }}$ & 0.8 & 2.0 \\
\hline$C N_{\text {CITe }}$ & 1.3 & 1.2 \\
\hline
\end{tabular}

Firstly, $g_{\text {TeTe}}(r)$ shows no significant differences between $\mathrm{Te}_{2} \mathrm{Cl}_{3}$ and $\mathrm{Te}_{3} \mathrm{Cl}_{2}$ in extrema positions up to $6 \AA$, and the computed $C N_{T e T e}$ is slightly and coherently weaker in $\mathrm{Te}_{2} \mathrm{Cl}_{3}$ than in $\mathrm{Te}_{3} \mathrm{Cl}_{2}$ (2.2 vs 2.8 respectively). We note that a similar trend, but with however lower values (1.46 vs 1.73 respectively - HE-XRD - Table 1), can been observed on the experimental side. Secondly, the first peak of $g_{T e C l}(r)$ is clearly sharper and its maximum shifted towards lower distance values in $\mathrm{Te}_{2} \mathrm{Cl}_{3}$ than in $\mathrm{Te}_{3} \mathrm{Cl}_{2}$. This combined to the fact that $\mathrm{CN}_{\mathrm{TeCl}}$ is more than two times larger in the former composition with respect to the latter one, suggests a coordination number change of $\mathrm{Cl}$ around Te upon the addition of chlorine, which translates into an overall increase for the tellurium atoms (4.2 vs 3.6 - Table 2). It must be pointed out that these latter results are also consistent with the evolution of the experimental $C N_{T e C l}$ and tellurium total coordination numbers (Table 1). Additionally, the $C N_{C l T e}$ values exceed 1, and are almost identical for the two compositions (1.3 and 1.2 for $\mathrm{Te}_{2} \mathrm{Cl}_{3}$ and $\mathrm{Te}_{3} \mathrm{Cl}_{2}$ ), indicating the presence of overcoordinated $\mathrm{Cl}$ in the glasses. It should be noted that the corresponding experimental values are in good agreement with the theoretical results, being equal to $\sim 1.2-1.3$ and $\sim 1.3-1.4$ for $\mathrm{Te}_{2} \mathrm{Cl}_{3}$ and $\mathrm{Te}_{3} \mathrm{Cl}_{2}$, respectively (Table 1). The presence of overcoordinated chlorine atoms is clearly a new feature with respect to the previous models used to describe those glasses. Indeed, in the most recent one derived from crystalline $\mathrm{Te}_{3} \mathrm{Cl}_{2}$ (Figure 1), only terminal $\mathrm{Cl}$ atoms were considered. Finally, it should be stressed that in both cases the $g_{\text {ClCl}}(r)$ functions show that there is no peak below $3 \AA$, which 
demonstrates the absence of $\mathrm{Cl}-\mathrm{Cl}$ bonds.

From the above-described partial radial distribution functions, the cutoff radii were determined in order to analyze the nature of the first coordination sphere of Te. We used 3.0 $\AA$ and $3.4 \AA$ for Te-Cl and Te-Te bonds respectively. Figure 11 contains the distribution diagrams of the tellurium coordination spheres. In $\mathrm{Te}_{3} \mathrm{Cl}_{2}$, Te atoms are mostly 3- and 4coordinated, with fractions of Te involved exclusively in homopolar bonds amounting to 27 and $\sim 12 \%$ of these coordination numbers respectively. If we evaluate the fraction of tellurium atoms connected to more than two other Te, irrespective of the presence of Te-Cl bonds, we obtain a value that exceeds $70 \%$. Taken as a whole, these findings suggest a clear departure from the 8-N rule for Te and indicate therefore a significant reticulation of the tellurium chains, this latter result being at variance with the model proposed on the basis of the $\mathrm{Te}_{3} \mathrm{Cl}_{2}$ crystal (Figure 1) in which chain branching was not considered. The reticulated tellurium network can be readily seen in Figure 12 from the selected snapshots for $\mathrm{Te}_{3} \mathrm{Cl}_{2}$ glass. Overcoordinated $\mathrm{Cl}$ atoms can also be detected in the simulation cell, showing that they are bridging Te units. These chlorine bridges lead to theoretical (and experimental) $C N_{C l-T e}>1$, and correspond also, as previously noted, to a novelty with respect to older TeX glass models. In the case of $\mathrm{Te}_{2} \mathrm{Cl}_{3}$ glass, the most probable Te coordination numbers are shifted upwards to 4 and 5, while 3-coordinated Te are only slightly lagging behind. The fraction of tellurium atoms bonded to 3 or more Te reduces to $\sim 40 \%$, which is not surprising given the higher percentage of $\mathrm{Cl}$ in that case. Conversely, with respect to $\mathrm{Te}_{3} \mathrm{Cl}_{2}$, a significant increase of the $\mathrm{Te}\left(\mathrm{TeCl}_{\mathrm{n}-1}\right), \mathrm{Te}\left(\mathrm{Te}_{2} \mathrm{Cl}_{\mathrm{n}-2}\right)$ and $\mathrm{Te}\left(\mathrm{Cl}_{\mathrm{n}}\right)$ unit fractions is found for $\mathrm{n}>3$. As will be discussed below, these entities will be of paramount importance to rationalize the solid-state NMR spectra (ssNMR) acquired on these glasses. 

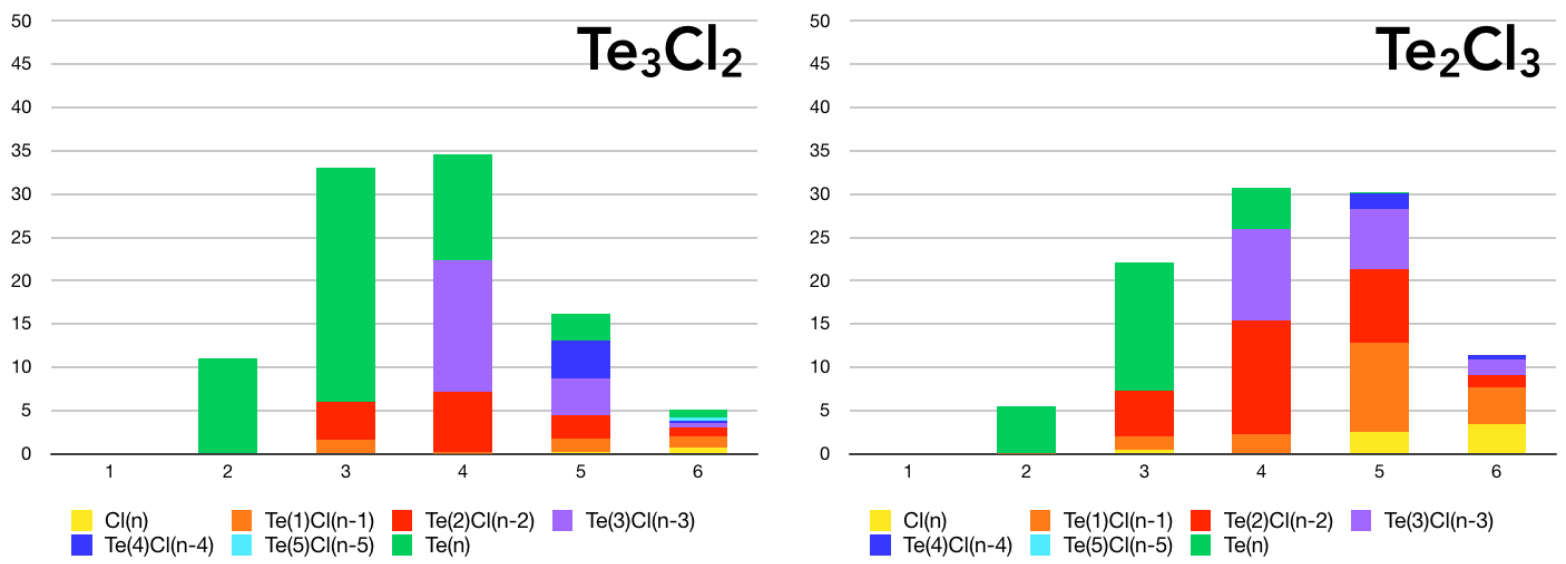

Figure 11. Distributions of Te coordination modes (\%) in $\mathrm{Te}_{3} \mathrm{Cl}_{2}$ (left) and $\mathrm{Te}_{2} \mathrm{Cl}_{3}$ (right). Total number of neighbours is in abscissa. Each color defines a given number of chalcogen atoms in the Te coordination sphere.
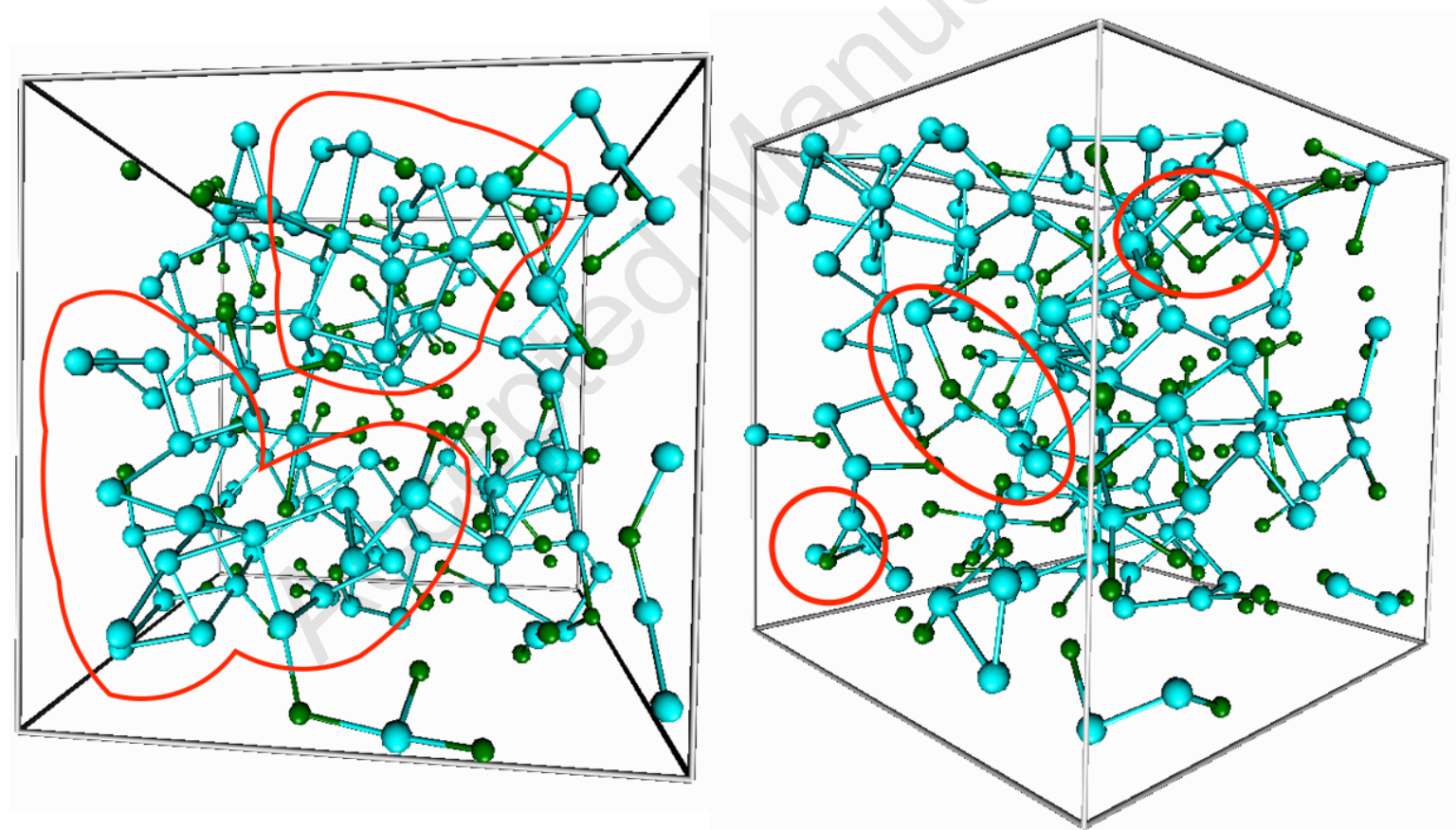

Figure 12. Selected snapshots taken from the $300 \mathrm{~K}$ trajectories of $\mathrm{Te}_{3} \mathrm{Cl}_{2}$ that show reticulated Te (blue) chains and bridging $\mathrm{Cl}$ (green).

In order to have access to a local probe of the atom specific environments in our $\mathrm{Te}_{2} \mathrm{Cl}_{3}$ and $\mathrm{Te}_{3} \mathrm{Cl}_{2}$ glasses, ${ }^{125} \mathrm{Te}$ ssNMR spectra were acquired so as to complement, at the local scale, the information collected using diffraction experiments (Figure 13). ${ }^{35 / 37} \mathrm{Cl}$ Solid-State NMR 
experiments were not attempted. Indeed, our GIPAW calculations led to excessively large $C_{Q}$ values (Figure S1), an order of magnitude higher than the values reported for silicate and aluminosilicate glasses by Sandland et $a l^{64}$. Concerning our ${ }^{125} \mathrm{Te}$ ssNMR spectra, the two lineshapes form a single Gaussian-type peak centred around 1200 ppm, having similar linewidths of $1500 \mathrm{ppm}$. It is immediately seen that, upon the addition of $\mathrm{Cl}$, a new sharp contribution appears at a higher chemical shift ( 1500 ppm), leading to a somewhat more complex structure for the $\mathrm{Te}_{2} \mathrm{Cl}_{3}$ signal.

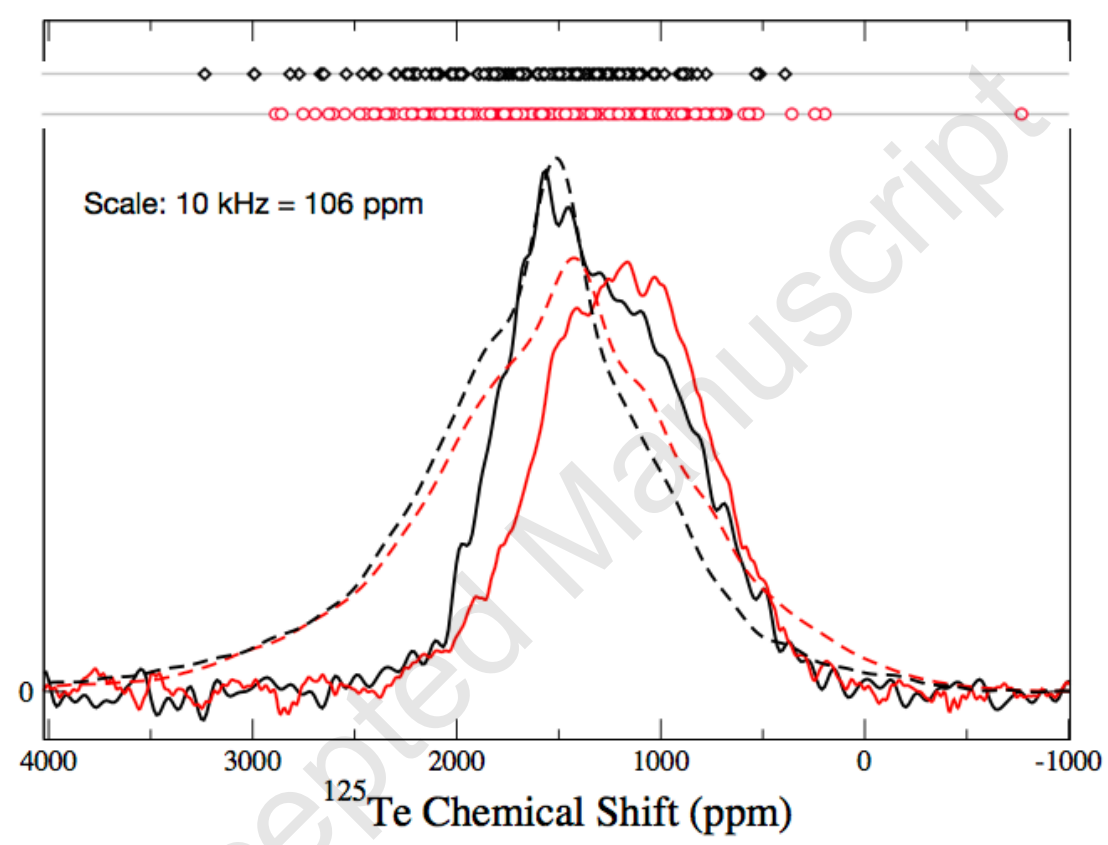

Figure 13. Experimental (solid line) and theoretical (dashed line) ${ }^{125} \mathrm{Te}$ NMR spectra of $\mathrm{Te}_{3} \mathrm{Cl}_{2}$ (red) and $\mathrm{Te}_{2} \mathrm{Cl}_{3}$ (black). A distribution of theoretical chemical shifts values is given at the top, as two lines of diamonds and circles, using the previously defined color coding. The measurements were performed on a $300 \mathrm{MHz}$ spectrometer at $10 \mathrm{kHz}$ MAS rotation, and the simulated spectra used a similar setup.

The simulated spectra, derived from the ${ }^{125}$ Te ssNMR parameters calculated within the GIPAW formalism on optimized snapshots of our in silico models, are also given in Figure 13. While the overall agreement with regards to the position of the spectra on the chemical 
shift scale is satisfying, we note that our spectra display larger linewidths by about 1000 ppm with respect to the experimental ones. We also note that the two theoretical lineshapes resemble lorentzians. These differences may be related to the fact that the theoretical anisotropy parameters are usually overestimated. Indeed, we obtain CSAs that can reach up to 2000 ppm, and averaged values of 945 and 922 ppm. Another factor may be related to the somewhat limited number of Te sites in our cells (160 and $240 \mathrm{Te}$ for $\mathrm{Te}_{2} \mathrm{Cl}_{3}$ and $\mathrm{Te}_{3} \mathrm{Cl}_{2}$, respectively), that precludes to a full match to the experimental shapes.

All in all, the agreement between theory and experiment can be considered as good, because despite the low structuration of the experimental spectra, we have succeeded in capturing the main features, i.e. a shift of the signal towards higher $\partial_{\text {iso }}$ values and the presence of a sharper peak, in the case of $\mathrm{Te}_{2} \mathrm{Cl}_{3}$.

An analysis of the simulated spectra on the basis of the various Te environments is given in Table 3.a). For 3-, 4- and 5-fold Te, corresponding to the most probable number of neighbors for the two glass compositions, we see that the calculated $<\partial_{\text {iso }}>$ for the four and five coordination modes, increases by more than $300 \mathrm{ppm}$ on going from $\mathrm{Te}_{3} \mathrm{Cl}_{2}$ to $\mathrm{Te}_{2} \mathrm{Cl}_{3}$. The 3fold Te appears to be affected by less than $100 \mathrm{ppm}$, most probably because in that case a large fraction of the chalcogen atoms form only Te-Te bonds (Figure 11). We therefore attribute the shift towards higher $\partial_{\text {iso }}$ values, observed for the $\mathrm{Te}_{2} \mathrm{Cl}_{3}$ ssNMR spectrum, to the electron withdrawing capability of the $\mathrm{Cl}$ atoms. Indeed, as previously noted, there is a drop in Te forming homopolar bonds in favor of heteropolar ones when the percentage of halogen increases (see Figure 11, for 4- and 5- coordinated Te). Turning to Table 3.b) that contains the ${ }^{125} \mathrm{Te}$ ssNMR parameters according to the number of $\mathrm{Cl}$ atoms bonded to $\mathrm{Te}$ atoms, independent of the total coordination number, gives additional clues as to the glassy structure. For the fractions exceeding $10 \%$ of the two compositions, i.e. for the first three lines of the table, an increase of at least $150 \mathrm{ppm}$ of the $<\partial_{\text {iso }}>$ can be calculated between $\mathrm{Te}_{3} \mathrm{Cl}_{2}$ and 
$\mathrm{Te}_{2} \mathrm{Cl}_{3}$. This fact reinforces the previous finding concerning the displacement of the $\mathrm{Te}_{2} \mathrm{Cl}_{3}$ spectrum towards high chemical shift value, with respect to $\mathrm{Te}_{3} \mathrm{Cl}_{2}$, due to the higher concentration of halogen at that composition. Moreover, given that the first line corresponds to Te atoms involved only in homopolar bonds, a long-range impact of the $\mathrm{Cl}$ atoms that extends beyond the first coordination sphere must be envisioned. Finally, one can note that the strongly chlorinated Te atoms (5 and $6 \mathrm{Cl}$ ), despite representing low fractions of the Te environments in the two glasses $\left(2.5 \%\right.$ and $10.6 \%$ respectively for $\mathrm{Te}_{3} \mathrm{Cl}_{2}$ and $\mathrm{Te}_{2} \mathrm{Cl}_{3}$ ), exhibit very low $<\mathrm{CSA}>$ values that are close to $200 \mathrm{ppm}$, while for the other environments they are in the 565-1233 ppm range. This result is also found for the hexacoordinated Te of Table 3.a), where the difference in $<\mathrm{CSA}>$ values with respect to the other units is at least equal to $345 \mathrm{ppm}$. We may relate these low $<\mathrm{CSA}>$ values to the rather high symmetries that one can expect for five and six-coordinated Te units, especially when $\mathrm{Cl}$ neighbours are almost exclusively present. In order to check for the impact of such highly coordinated-low CSA structural units, they have been identified in the theoretical ${ }^{125}$ Te NMR spectrum of $\mathrm{Te}_{2} \mathrm{Cl}_{3}$ reported in Figure 14. It is immediately seen, that the sharp peak present around 1500 ppm in the experimental lineshape of the $\mathrm{Te}_{2} \mathrm{Cl}_{3}$ glass (Figure 13) can therefore be confidently attributed to the formation of 5 and 6-coordinated Te units, consisting mostly of halogen first neighbours. It should be pointed out that, so far, such structural units where not considered in earlier glass models based on chlorinated Te chains. At most only $\mathrm{Te}-\mathrm{TeCl}_{4}$, ending chains, were taken into account. 
Table 3. Averaged isotropic chemical shifts (ppm) and anisotropies (ppm) for the different coordination modes of Te (a) and for the different number of linked $\mathrm{Cl}$ (b), in $\mathrm{Te}_{3} \mathrm{Cl}_{2}$ (left) and $\mathrm{Te}_{2} \mathrm{Cl}_{3}$ (right). Quantities of each environment are given in number and in percent.

(a)

\begin{tabular}{|c|c|c|c|c|c|c|c|c|}
\hline n-fold & \multicolumn{2}{|l|}{$\mathrm{Te}_{3} \mathrm{Cl}_{2}$} & \multicolumn{2}{l|}{$\mathrm{Te}_{2} \mathrm{Cl}_{3}$} \\
\hline & $<$ iso $>$ & $<$ CSA $>$ & quantity & $\%$ & $<$ iso $>$ & $<$ CSA $>$ & quantity & $\%$ \\
\hline 2 & 1869 & 1009 & 25 & 10.4 & 1819 & 1326 & 9 & 5.6 \\
\hline 3 & 1575 & 1109 & 76 & 31.7 & 1660 & 1173 & 30 & 18.8 \\
\hline 4 & 1323 & 909 & 84 & 35.0 & 1639 & 1101 & 53 & 33.1 \\
\hline 5 & 1215 & 843 & 41 & 17.1 & 1520 & 731 & 46 & 28.8 \\
\hline 6 & 1143 & 449 & 14 & 5.8 & 1235 & 386 & 22 & 13.8 \\
\hline total & 1431 & 945 & 240 & 100.0 & 1563 & 922 & 160 & 100.0 \\
\hline
\end{tabular}

(b)

\begin{tabular}{|c|c|c|c|c|c|c|c|c|}
\hline $\mathrm{n} \mathrm{Cl}$ & \multicolumn{2}{|l|}{$\mathrm{Te}_{3} \mathrm{Cl}_{2}$} & \multicolumn{2}{l|}{$\mathrm{Te}_{2} \mathrm{Cl}_{3}$} \\
\hline & $<$ iso $>$ & $<$ CSA $>$ & quantity & $\%$ & $<$ iso $>$ & $<$ CSA $>$ & quantity & $\%$ \\
\hline $0 \mathrm{Cl}$ & 1478 & 1061 & 123 & 51.3 & 1627 & 1171 & 42 & 26.3 \\
\hline $1 \mathrm{Cl}$ & 1353 & 889 & 62 & 25.8 & 1543 & 1233 & 23 & 14.4 \\
\hline $2 \mathrm{Cl}$ & 1445 & 879 & 35 & 14.6 & 1614 & 1007 & 42 & 26.3 \\
\hline $3 \mathrm{Cl}$ & 1425 & 606 & 7 & 2.9 & 1458 & 806 & 15 & 9.4 \\
\hline $4 \mathrm{Cl}$ & 1247 & 695 & 7 & 2.9 & 1584 & 565 & 21 & 13.1 \\
\hline $5 \mathrm{Cl}$ & 1381 & 216 & 4 & 1.7 & 1335 & 230 & 10 & 6.3 \\
\hline $6 \mathrm{Cl}$ & 1410 & 211 & 2 & 0.8 & 1424 & 216 & 7 & 4.4 \\
\hline total & 1430 & 945 & 240 & 100.0 & 1563 & 922 & 160 & 100.0 \\
\hline
\end{tabular}




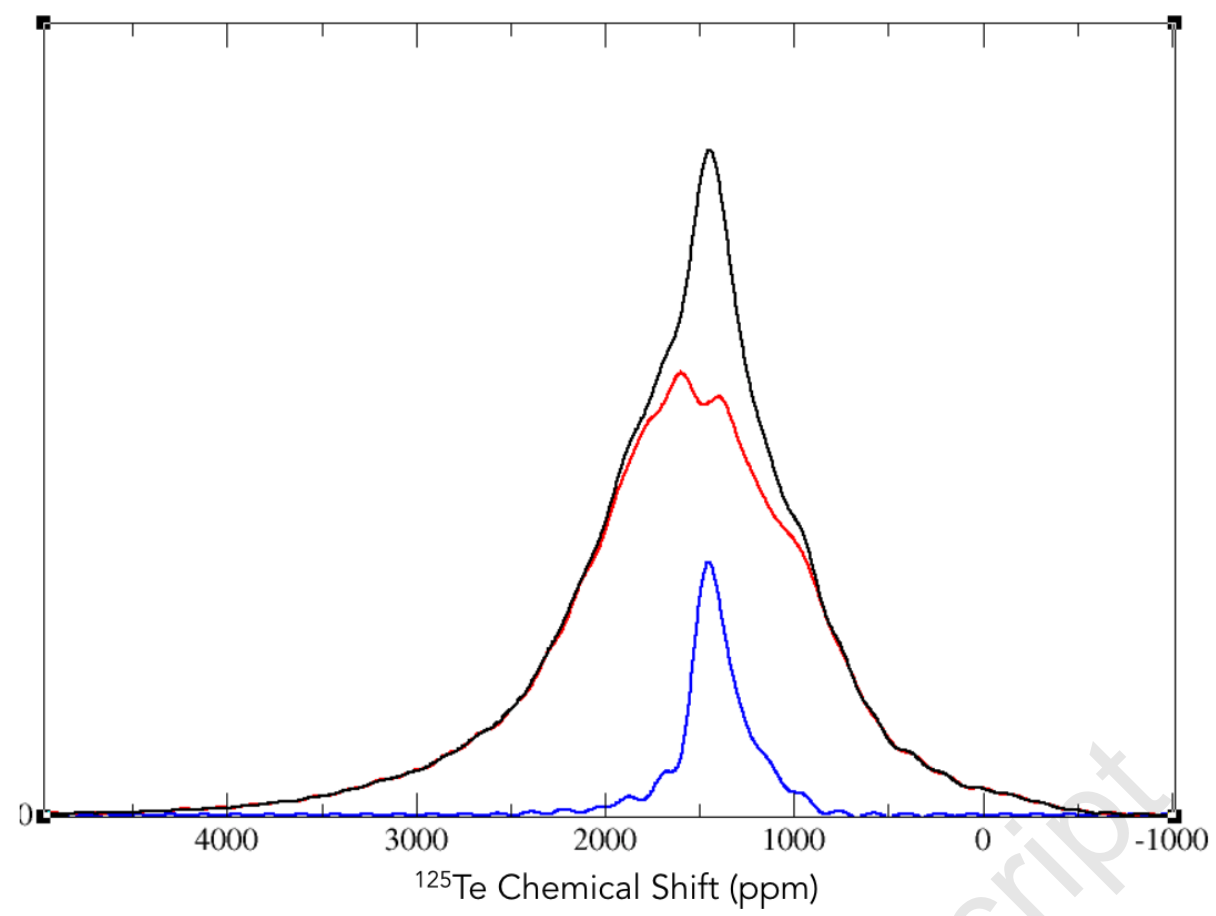

Figure 14. Theoretical ${ }^{125} \mathrm{Te}$ NMR spectrum of $\mathrm{Te}_{2} \mathrm{Cl}_{3}$ glass (black) emulated for a $300 \mathrm{MHz}$ spectrometer at $10 \mathrm{kHz}$ MAS rotation. The contribution of $\mathrm{TeCl}_{6}$ and $\mathrm{TeCl}_{5} \mathrm{X}(\mathrm{X}=\mathrm{Te}$ or nothing) is shown in blue and the contribution of all other environments is in red.

\section{Conclusion}

A combination of X-ray, neutron, ${ }^{125} \mathrm{Te}$ ssNMR experiments and molecular dynamics calculations has been presented in order to deepen the knowledge on the atomic-scale organization of the Te-Cl glass materials that are considered as prototypical chalcogenide glasses. With respect to the previous model used to describe these glasses, which was relied heavily on the $\mathrm{Te}_{3} \mathrm{Cl}_{2}$ crystalline structure, both experimental and theoretical data agree to the fact that the room temperature structure of these amorphous materials is not based on $\mathrm{Cl}$ terminated tellurium chains of variable lengths. Indeed, our results suggest a reticulated network of chalcogen atoms, with a number of chlorine atoms bonded in a bridging fashion. Thus, a significant departure from the $8-\mathrm{N}$ rule can be noted in these glasses for a large fraction of the two types of atoms. We also showed that the coordination mode of Te tends to 
increase with the amount of $\mathrm{Cl}$, leading to the formation of rather high symmetry (pseudopyramidal or pseudo-octahedral) units having a strongly chlorinated coordination sphere. This is reminiscent of the crystal structure of $\mathrm{TeCl}_{4}$, that may be considered as consisting of deformed $\mathrm{TeCl}_{6}$-like octahedra. We have also demonstrated that these structural units found in the glasses, exhibit low CSA values and that they are therefore responsible of the sharp peak feature that could be detected on the experimental ${ }^{125} \mathrm{Te}$ NMR spectrum of $\mathrm{Te}_{2} \mathrm{Cl}_{3}$. Finally, we observed a drift of the NMR signal towards high chemical shift values, which is consistent with the chlorine fraction in the material and the electron withdrawing capabilities of this element.

\section{Conflicts of interest}

There are no conflicts to declare.

\section{Acknowledgments}

The synchrotron x-ray scattering experiments were performed both at the BL04B2 in the SPring-8 with the approval of the Japan Synchrotron Radiation Research Institute (JASRI) (Proposal Number 2011B1565) and at the Advanced Photon Source, Argonne National Laboratory with a partial support of the Office of Basic Energy Sciences, U.S. Department of Energy under contract No. DE-AC02-06CH1135. The authors are also grateful to Dr. B. Beuneu for the helpful assistance and useful discussions for neutron diffraction experiments at the Laboratory Leon Brillouin (LLB). This work was also granted access to the HPC resources of IDRIS, CINES and TGCC under the allocation 2016-086045 made by GENCI (Grand Equipement National de Calcul Intensif). 
Supporting Information. Graph of GIPAW calculated $\mathrm{C}_{\mathrm{Q}}$ values for all sites of the $\mathrm{Te}_{3} \mathrm{Cl}_{2}$ and $\mathrm{Te}_{2} \mathrm{Cl}_{3}$ glass models - Comparison of MAS and static ${ }^{125} \mathrm{Te}$ NMR spectra on $\mathrm{Te}_{3} \mathrm{Cl}_{2}$ glass

\section{References}

\footnotetext{
${ }^{1}$ Zhang, X. H.; Bureau, B.; Lucas, P.; Boussard-Plédel, C.; Lucas, J. Glasses for seing beyond visible. Chem. Eur. J. 2008, 14, 432-442.

2 Zakery, A.; Elliott, S. R. Optical properties and applications of chalcogenide glasses : a review. J. Non-Cryst. Solids 2003, 330, 1-12.

${ }^{3}$ Seddon, A. B. Chalcogenide glasses : a review of their preparation, properties and applications. J. Non-Cryst. Solids 1995, 184, 44-50.

${ }^{4}$ Lucas, J.; Zhang, X. H. The tellurium halide glasses. J. Non-Cryst. Solids 1990, 125, 1-16.

${ }^{5}$ Borisova, Z. U. Glassy Semiconductors. Plenum Press, New York 1981

${ }^{6}$ Pendas, A. M.; Costales, A.; Luana, V. Ions in crystals: the topology of the electron density in ionic materials. III. Geometry and ionic radii. J. Phys. Chem. B 1998, 102, 6937.
}

7 Wells, J.; Bresser, W. J.; Boolchand, P.; Lucas, J. Medium range structure in a network glass established by a local probe. J. Non-Cryst. Solids 1996, 195, 170.

${ }^{8}$ Kniep, R.; Mootz, D.; Rabenau, A. Structural Investigations of Tellurium Subhalides: Modified Tellurium Structures. Angew. Chem. Int., 1973, 12, 499-500.

9 Suehara, S.; Noguera, O.; Aizawa, T.; Sasaki, T.; Lucas, J. Ab initio calculation of chains structures in chalcohalide glasses. J. Non-Cryst. Solids 2008, 354, 168-172.

10 Zotov, N.; Beck, J.; Knopp, B.; Kirfel, A. Structural Study of Amorphous Tellurium(II) Halides TeCl ${ }_{2-\mathrm{x}} \mathrm{I}_{\mathrm{x}}(\mathrm{x}=$ 0.5, 0.1): X-ray Diffraction and Reverse Monte Carlo Simulations. Inorg. Chem. 2007, 46, 4286-4292.

${ }^{11}$ Bouzid, A.; Pizzey, K. J.; Zeidler, A.; Ori, G.; Boero, M.; Massobrio, C.; Klotz, S.; Fischer, E. H.; Bull C. L.; Salmon, P. S. Pressure-induced structural changes in the network-forming isostatic glass GeSe e $_{4}$ an investigation by neutron diffraction and first-principles molecular dynamics. Phys. Review B 2016, 93, 14202.

12 Voleská, I.; Akola, J.; Jóvári, P.; Gutwirth, J.; Wágner, T.; Vasileiadis, T.; Yannopoulos, S. N.; Jones, R. O. Structure, electronic, and vibrational properties of glassy $\mathrm{Ga}_{11} \mathrm{Ge}_{11} \mathrm{Te}_{78}$ : Experimentally constrained density functional study. Phys. Rev. B 2012, 86, 94108.

${ }^{13}$ Micoulaut, M.; Coulet, M.V.; Piarristeguy, A.; Johnson, M. R.; Cuello, G. J.; Bichara, C.; Raty, J. Y.; FloresRuiz, H.; Pradel, A. Effect of concentration in Ge-Te liquids: A combined density functional and neutron scattering study. Phys. Rev. B 2014, 89, 1-13.

${ }^{14}$ Kaseman, D. C.; Hung, I.; Lee, K.; Kovnir, K.; Gan, Z.; Aitken, B.; Sen, S. Tellurium speciation, connectivity, and chemical order in $\mathrm{As}_{\mathrm{x}} \mathrm{Te}_{100-\mathrm{x}}$ glasses: results from two-dimensional ${ }^{125} \mathrm{Te}$ NMR spectroscopy. J. Phys. Chem. B 2015, 119, 2081-2088.

15 Yildirim, C.; Micoulaut, M.; Boolchand, P.; Kantor, I.; Mathon, O. ; Gaspard, J.-P.; Raty, J.-Y. Universal amorphous-amorphous transition phase in $\mathrm{Ge}_{\mathrm{x}} \mathrm{Se}_{100-\mathrm{x}}$ glasses under pressure. Scientific Reports 2016, 6, 27317.

${ }^{16}$ Bouëssel du Bourg, L.; Roiland, C.; Le Pollès, L.; Deschamps, M.; Boussard-Plédel, C.; Bureau, B.; Furet, E. Impact of Te on the structure and ${ }^{77}$ Se NMR spectra of Se-rich Ge-Te-Se glasses : a combined experimental and computational investigation. Phys. Chem. Chem. Phys. 2015, 17, 29020-29026.

17 Sykina, K.; Bureau, B.; Le Pollès, L.; Roiland, C.; Deschamps, M.; Pickard, C. J.; Furet, E. A combined ${ }^{77}$ Se NMR and molecular dynamics contribution to the structural understanding of the chalcogenide glasses. Phys. Chem. Chem. Phys. 2014, 16, 17975.

18 Ambroise, J.-P.; Bellissent, R. Neutron scattering determination of local order in amorphous and liquid systems using a position sensitive detector. Rev. Phys. Appl. 1984, 19, 731-734.

19 Ellison, A. J. G.; Crawford, R. K.; Montague, D. G.; Volin K. J.; Price D. L. The new glass liquid and amorphous materials diffractometer (GLAD) at IPNS. J. Neutron Res. 1993, 1, 61-70

${ }^{20}$ Susman, S.;Volin, K.J.; Montague, D.G.; Price D.L. The Structure of Vitreous and Liquid GeSe2: A Neutron Diffraction Study. J. Non-Cryst. Solids 1990, 125, 168-180 
21 Dejus R.J., Susman S., Volin K.J., Montague D.G., Price D.L. Structure of Vitreous of Ag-Ge-Se. J. NonCryst. Solids 1992, 143, 162-180

22 Urquidi, J.; Benmore, C. J.; Neuefeind, J.; Tomberli, B. ISOMER-X: a program for the analysis of highenergy X-ray diffraction experiments. J. Appl. Crystallogr. 2003, 36, 368.

${ }^{23}$ Sakida, S.; Hayakawa, S.; Yoko, T. Part $1 .{ }^{125}$ Te NMR study of tellurite crystals J. Non-Cryst. Solids 1999, 243, 1-12.

${ }^{24}$ Car, R.; Parrinello, M. Unified approach for molecular dynamics and density-functional theory. Phys. Rev. Lett. 1985, 55, 2471-2474.

25 Marx, D.; Hutter, J. in Ab-initio Molecular Dynamics: Theory and Implementation, ed. J. Grotendorst, Forschungszentrum Julich. ch. 13, 2000, 301-449

${ }^{26}$ Perdew, J. P.; Burke, K.; Ernzerhof, M. General gradient approximation made simple. Phys. Rev. Lett. 1996,

27 Troullier, N.; Martins, J. L. Efficient pseudo-potentials for plane-wave calculation. II. Operators for fast iterative diagonalization. Phys. Rev. B 1991, 43, 8861-8869.

28 Troullier, N.; Martins, J. L. Efficient pseudo-potentials for plane-wave calculation. Phys. Rev. B 1991, 43 , 1993-2006.

${ }^{29}$ Nosé, S. A molecular dynamics method for simulations in the canonical ensemble. Mol. Phys. 1984, 52, 255268.

30 Hoover, W. G. Canonical dynamics : equilibrium phase-space distributions. Phys. Rev. A: At., Mol., Opt. Phys. 1985, 31, 1695-1697.

31 Grimme, S. Semi empirical GGA-type density functional constructed with a long-range dispersion correction. J. Comp. Chem. 2006, 27, 1787.

32 Pickard, C. J.; Mauri, F. All-electron magnetic response with pseudopotentials: NMR chemical shifts. Phys. Rev. B 2001, 63, 245101.

33 Yates, J. R.; Pickard, C. J.; Mauri, F. Calculation of NMR chemical shifts for extended systems using ultrasoft pseudopotentials. Phys. Rev. B 2007, 76, 024401.

34 Clark, S.; Segall, M.; Pickard, C. J.; Hasnip, P.; Probert, M.; Refsonand, K.; Payne, M. First principles methods using CASTEP. Zeitschriftfür Kristallographie 2005, 220, 567-570.

${ }^{35}$ Haeberlen, U.; High Resolution Nmr in Solids: Selective Averaging, Academic Press, New York, 1976

36 Bak, M.; Rasmussen, J. T.; Nielsen, N. C. SIMPSON : a general simulation program for solid-state NMR spectroscopy. J. Magn. Reson. 2011, 213, 366-400.

37 Bychkov, E.; Miloshova, M.; Price, L.; Benmore, C. J.; Lorriaux, A. Short, intermediate and mesoscopic range order in sulfur-rich binary glasses. J. Non-Cryst. Solids 2006, 352, 63-70.

38 Boolchand, P.; Georgiev, D. G.; Qu,T.; Wang, F.; Cai, L.; Chakravarty, S. Nanoscale phase separation effects near $\mathrm{r}=2.4$ and 2.67, and rigidity transitions in chalcogenide glasses. C. R. Chimie 2002, 5, 713.

${ }^{39}$ Giridhar, A.; Mahadevan, S; J. Non-Cryst. Solids 1992, 151, 245-252.

40 Bureau, B.; Boussard-Plédel, C.; Le Floch, M.; Troles, J.; Smektala, F.; Lucas, J. Selenium-Tellurium Sequences in Binary Glasses as Depicted by ${ }^{77}$ Se and ${ }^{125}$ Te NMR. J. Phys Chem B 2005, 109, 6130-6135.

${ }^{41}$ Elliott, S. R. Medium-range structural order in covalent amorphous solids, Nature 1991, 354, 445-452.

42 Salmon, P. S. Real space manifestation of the first sharp diffraction peak in the structure factor of liquid and glassy materials, Proc. R. Soc. London, Ser. 1994, A 445, 351-365

43 Moss, S. C.; Price, D. L. in Physics of Disordered Materials, edited by D. Adler, H. Fritzsche, and S. R. Ovshinsky (Plenum, New York, 1985)

${ }^{44}$ Wicks, J. D.; McGreevy, R. L.; Börjesson, L. A network problem: modelling alkali-silicate glasses with RMC, Phase Transitions 1997, 61,195-213.

45 Petri, I. ; Salmon, P. S. ; Fischer, H. E. Defects in a disordered world: the structure of glassy GeSe 2 , Phys. Rev. Lett. 2000, 84, 2413-2416.

${ }^{46}$ Bychkov, E.; Benmore, C. J.; Price, D. L. Compositional changes of the first sharp diffraction peak in binary selenide glasses, Phys. Rev. B: Condens. Matter Mater. Phys. 2005, 72, 172107. 
47 Vashishta, P.; Kalia, R. K.; Antonio, G. A.; Ebbsjö, I. Atomic correlations and intermediate-range order in molten and amorphous $\mathrm{GeSe}_{2}$, Phys. Rev. Lett. 1989, 62, 1651-1654

${ }^{48}$ Vashishta, P. ; Kalia, R. K.; Ebbsjö, I. Structural correlations and phonon density of states in GeSe ${ }_{2}$ : A molecular-dynamics study of molten and amorphous states, Phys. Rev. B: Condens. Matter Mater. Phys. 1989, 39, 6034-6047

${ }^{49}$ Buss,B.; Krebs, B. The crystal structure of tellurium tetrachloride. Inorg. Chem. 1971, 10, 2795-2800.

${ }^{50}$ Le Coq, D.; Bytchkov, A.; Honkimäki, V.; Beuneu, B.; Bychkov, E. Neutron and X-ray diffraction studies of $\mathrm{TeCl}_{4}$ and $\mathrm{TeBr}_{4}$ liquids, J. Non-Cryst. Solids 2008, 354, 259-262

51 Stevenson, D. P.; Schomaker, V. An electron diffraction investigation of the molecular structure of tellurium tetrachloride, J. Amer. Chem. Soc. 1940, 62, 1267-1270.

52 Kovács, A.; Martinsen, K.-G.; Konings, R. J. M. Gas-phase vibrational spectrum and molecular geometry of $\mathrm{TeCl}_{4}$, J. Chem; Soc., Dalton Trans. 1997, 1037-1042.

53 Hannon, A. C. XTAL: a program for calculating interatomic distances and coordination numbers for model structures, Rutherford Appleton Laboratory Report. 1993, RAL-93-063..

${ }^{54}$ Bosi, P.; Cilloco, F.; Ricci, F. P.; Sacchetti, F. The structure of liquid chlorine, Mol. Phys. 1983, 49, 567-581

55 Donohue, J.; Goodman, S. H. Interatomic distances in solid chlorine. Acta Cryst. 1965, 18, 568-569

56 Powell, B. M.; Heal, K. M.; Torrie B. H. The temperature dependence of the crystal structures of the solid halogens, bromine and chlorine, Mol. Phys. 1984, 53, 929-939

57 Alemi, A.; Soleimani, E.; Starikova, Z. A. The novel route for synthesis of tellurium tetrachloride, and redetermination of its structure at lower temperature by X-ray crystallography, Acta Chim. Sloven. 2000, 47, 8998

58 Troyanov, S. I.; Krahl, T.; Kemnitz, E. Crystal structures of $\mathrm{GaX}_{3}(\mathrm{X}=\mathrm{Cl}, \mathrm{Br}, \mathrm{I})$ and $\mathrm{AlI}_{3}$. Z. Kristal. 2004, 219, 88-92.

59 Petrov, V. M.; Giricheva, N. I.; Girichev, G. V.; Titov, V. A.; Chusova, T. P. Electron diffraction study of saturated vapor of gallium trichloride: vapour composition and structure of molecular species, J. Struct. Chem. 1992, 32, 498-502.

${ }^{60}$ Haaland, A.; Hammel, A.; Martinsen, K.-G.; Tremmel, J.; Volden, H. V. Molecular structures of monomeric gallium trichloride, indium trichloride and lead tetrachloride by gas electron diffraction, J. Chem. Soc. Dalton Trans. 1992, 14, 2209-2214

${ }^{61}$ Le Coq, D.; Beuneu, B.; Bychkov, E. Structure of $\mathrm{Te}_{1-\mathrm{x}} \mathrm{Cl}_{\mathrm{x}}$ liquids. AIP Conf. Proc. 2008, 982, 712-716.

${ }^{62}$ Kohzu, K.; Taketoshi, K. Structural study on amorphous tellurium using reverse Monte Carlo simulation. Jpn. J. Appl. Phys. 2002, 41, 6084.

63 Bichara, C.; Raty, J.-Y.; Gaspard, J.-P. Structure and bonding in liquid tellurium. J. Non-Cryst. Solids 1996, 205-207, 361-364.

${ }^{64}$ T.O. Sandland, L. Du, J. F. Stebbins, J. D. Webster Structure of Cl-containing silicate and aluminosilicate glasses: A ${ }^{35} \mathrm{Cl}$ MAS_NMR study Geochimica et Cosmochimica Acta, 2004, 68, 5059-5069. 


\section{For Table of Contents Only}

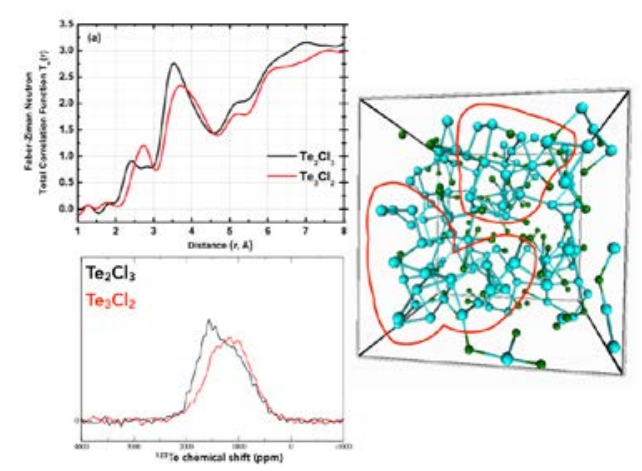

\section{Synopsis}

X-ray, Neutron, ${ }^{125}$ Te solid-state NMR experiments, Car-Parrinello simulations and GIPAW calculations have been combined to give access to the atomic-scale organization of glasses in the Te-Cl system 


\section{Supporting Information :}

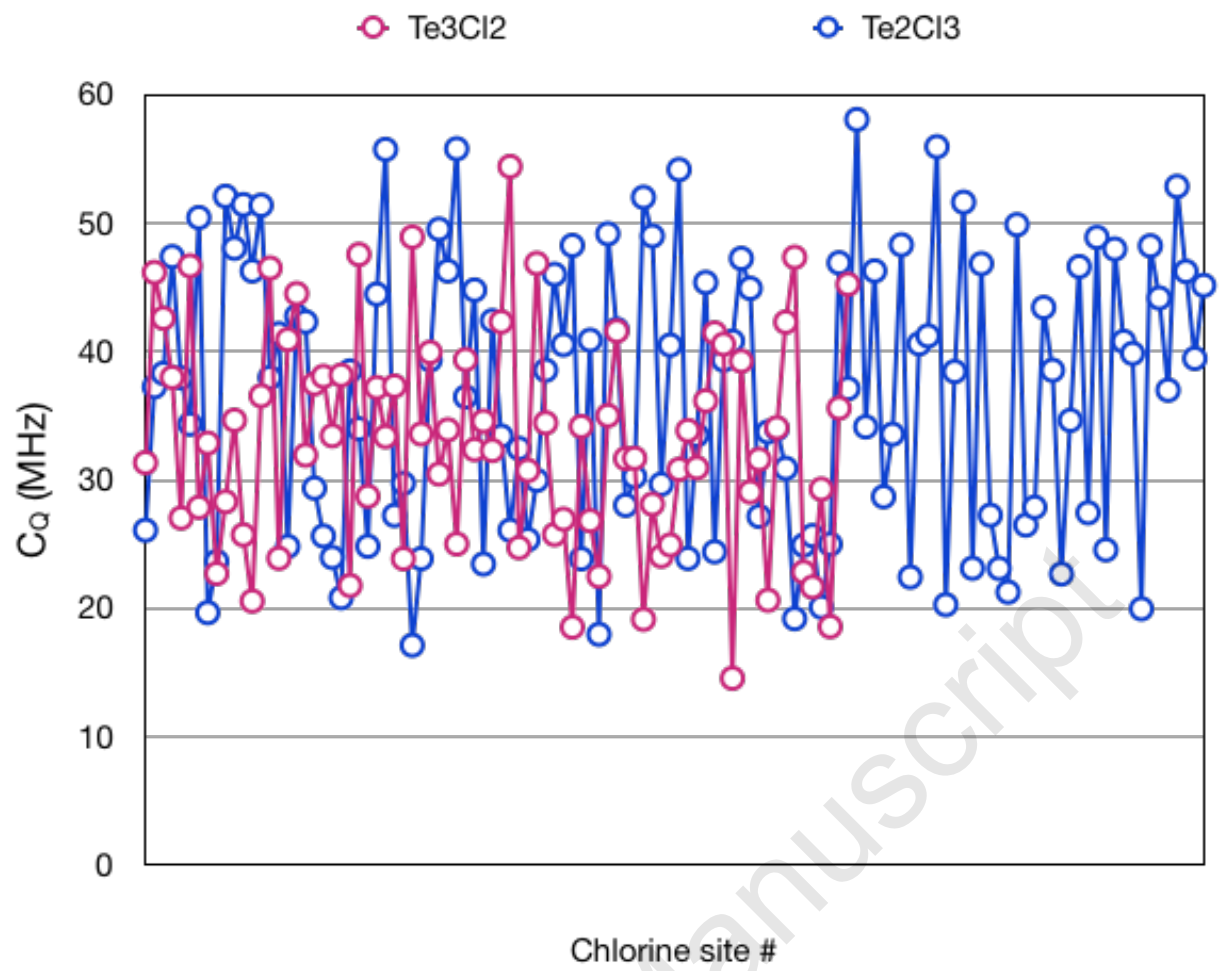

Figure S1 : Graph of the calculated $C_{Q}$ values for all chlorine sites in the $\mathrm{Te}_{3} \mathrm{Cl}_{2}$ (red - 80 sites) and $\mathrm{Te}_{2} \mathrm{Cl}_{3}$ (blue - 120 sites) glass models.

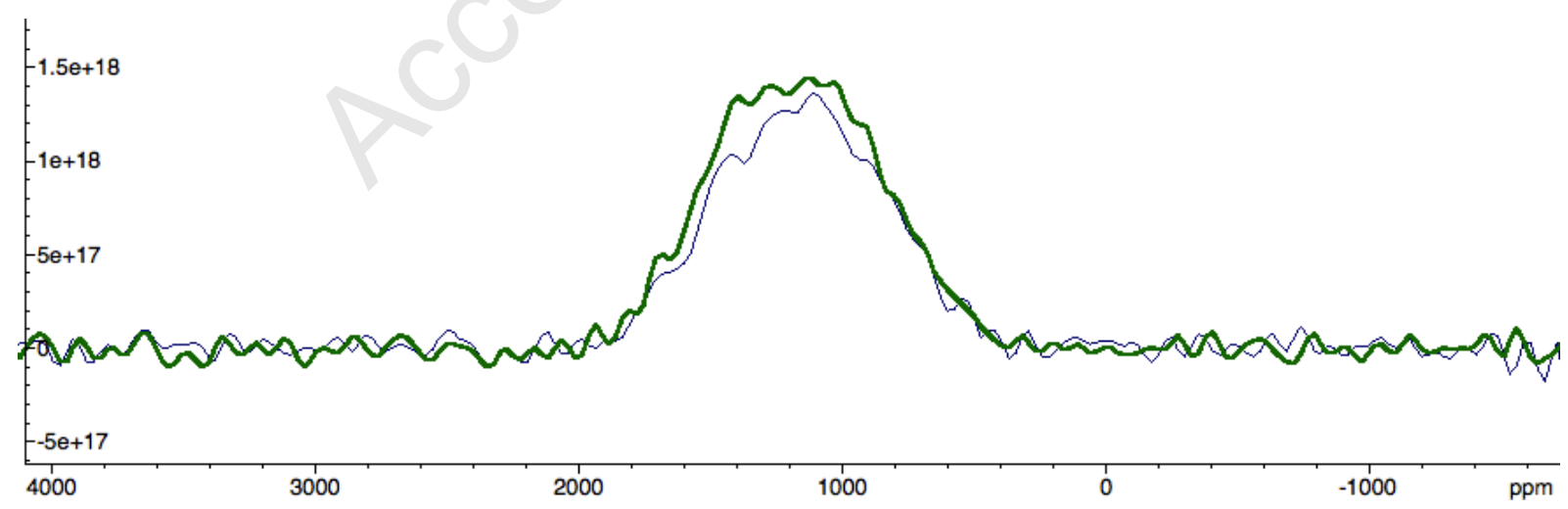

Figure S2 : ${ }^{125} \mathrm{Te} \mathrm{NMR}$ on $\mathrm{Te}_{3} \mathrm{Cl}_{2}$ glass at $7 \mathrm{~T}$, comparison of static (green) and MAS at 10 $\mathrm{kHz}$ spinning rate (blue) 